Commun. math. Phys. 3, 151-180 (1966)

\title{
Invariant States in Statistical Mechanics
}

\author{
Daniel Kastler* and Derek W. Robinson* \\ University of Aix-Marseille \\ Received April 1, 1966
}

\begin{abstract}
Mathematically we consider a $C^{*}$-algebra $\mathfrak{A}$, acted upon by the group $\mathbf{T}$ of space-translations, which has an asymptotic abelian property. We analyse invariant states over $\mathfrak{A}$. Physically this programme can be considered as a kinematical study of equilibrium states in statistical mechanics. Each invariant state can be uniquely decomposed into elementary invariant states ( $E$-states). These elementary states have, amongst other characteristics, the physical property that space-averages of local observables are constants in the corresponding representations. In an $E$-state the discrete spectrum $\mathrm{S}_{D}$ of space-translations is additive which gives rise to the classification $E_{\mathrm{I}}, E_{\mathrm{II}}$, and $E_{\mathrm{III}}$ corresponding to the three possibilities that $\mathbf{S}_{D}$ contains one point, a lattice of points, or a set with accumulation points. An $E_{I I}$-state can be uniquely decomposed into states ( $L$-states) having a symmetry with respect to a closed subgroup $\mathbf{T}_{L}$ of space-translations $\left(\mathbf{S}_{D}\right.$ and $\mathbf{T}_{L}$ are reciprocal lattices). $L$-states have properties with respect to $\mathbf{T}_{L}$ analogous to the properties of $E_{\mathrm{I}}$-states with respect to $\mathbf{T}$. The decomposition into $L$-states is the inverse process of 'homogenizing' a lattice state by smearing it over a lattice distance. The mathematical methods which we employ have more general applications.
\end{abstract}

\section{Introduction}

In a recent paper [1] with $\mathrm{S}$. DopLICHER, which we refer to as DKR, we among other things partially analysed a class of states which we consider to be suitable as equilibrium states in statistical mechanics. It is the purpose of the present paper to generalise and extend this analysis. As our approach to statistical mechanics is rather different from the traditional one let us first briefly state our point of view. In the usual formulation one considers first the microscopic problem of a finite number $N$ of particles in a box of volume $V$ and then considers the macroscopic limit $V \rightarrow \infty$ with the mean density $N / V$ held fixed. Instead of following this procedure we wish to consider directly a description of the macroscopic or infinite, system. In order to understand our procedure it is worthwile to recall a few of the salient features of the microscopic approach. If we consider the quantum mechanical case the description of $N$ particles in volume $V$ is given kinematically by the Fock representation of the canonical commutation, or anti-commutation, relations (for further details see, for instance, [2], [3], [4]) and dynamically by speci-

* Postal Address: Physique Theorique, Faculté des Sciences, Place Victor-Hugo Marseille - France.

11 Commun. math. Phys., Vol. 3 
fication of the Hamiltonian describing the interaction of the particles. In the limit of an infinite volume the simplicity of this description is lost and other states or representations of the gauge invariant subalgebra of the commutation (anti-commutation) relations occur which are not unitarily equivalent to the Fock representation; the particular state which occurs is determined by the interaction i.e. the Hamiltonian [5]. This complication is a consequence of the fact that in the macroscopic system we have an infinite number of degrees of freedom. However to compensate this complication there is an added simplification in the description of the infinite system because the limit states have the property of invariance under the group of space translations. Thus the kinematic description of equilibrium states in quantum statistical mechanics should be given by a discussion of the translationally invariant states of the gauge invariant subalgebra of the commutation (anti-commutation) relations. In the present paper we will consider a more general problem and analyse the translationally invariant states over an algebra $\mathfrak{A}$ which has the structure

$$
\|[A(x), B]\| \underset{|x|=\infty}{\longrightarrow} 0, \quad A, B \in \mathfrak{Z} .
$$

This general approach has the advantage of not only covering the quantum mechanical case but also covering abelian algebras which are of interest in classical statistical mechanics.

The results of our analysis are as follows. Any invariant state over $\mathfrak{A}$ has a unique decomposition (theorem 2 ) into invariant states of a special type which we call $E$-states. These $E$-states were already considered in DKR and they can be characterised by a number of equivalent conditions (theorem 3) of which we mention the following three. Firstly if $\pi(A)$ and $U(x)$ are the representations, determined by an $E$-state, of $\mathfrak{A}$ and the group $\mathbf{T}$ of space-translations respectively, then the set of operators $\pi(\mathfrak{A}) \cup U(\mathbf{T})$ is irreducible in the representation space $\mathfrak{H}$. Secondly, in $\mathfrak{G}$ there is a unique translationally invariant vector $\Omega$. Thirdly, we have

$$
\lim _{V \rightarrow \infty} \frac{1}{V} \int_{V} d x(\psi|\pi(A(x))| \varphi)=(\Omega|\pi(A)| \Omega)(\psi \mid \varphi)
$$

for all $A \in \mathfrak{A}$ and $\psi, \varphi \in \mathfrak{H}$. This last condition expresses the physical result that the global average of a local observable exists and is a constant in the representation determined by an $E$-state. Further analysis (theorem 4 ) shows that $E$-states can be divided into three classes $E_{\text {I }}$, $E_{\mathrm{II}}$ and $E_{\mathrm{III}}$-states, where the classification is with respect to the discrete eigenstates of $U(x)$. In an $E_{\mathrm{I}}$-state there is one and only one discrete

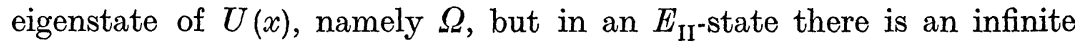
number of discrete eigenstates occurring with a lattice structure. In an

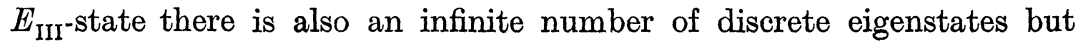


they occur in a dense manner making it impossible to define a lattice distance. Next (theorem 5), we show that an $E_{\mathrm{II}}$-state has a unique decomposition into states which are invariant under a closed subgroup $\mathbf{T}_{L}$ of space-translations and which we call $L$-states. These latter lattice type states can also be characterised by a number of equivalent conditions (theorem 6) which are similar to those characterising $E_{\mathrm{I}}$-states: if $U_{L}\left(x_{L}\right)$ is the representation of $\mathbf{T}_{L}$ then the set of operators $\pi(\mathfrak{Q}) \cup U_{L}\left(\mathbf{T}_{L}\right)$ is irreducible, there is a unique vector $\Omega_{L}$ which is invariant under $U_{L}\left(\mathbf{T}_{L}\right)$, and a condition similar to (2) holds but the mean value is taken over lattice points. The $L$-states are analogous to $E_{\mathrm{I}}$-states insofar that they give rise to only one discrete eigenstate of $\mathbf{T}_{L}$, namely $\Omega_{L}$. We thus see that the analysis of invariant states delivers in a unique and natural manner states of a homogeneous nature ( $E_{\mathrm{I}}$-states), states of a periodic nature ( $L$-states) in which the space-translation symmetry is broken and states of the mysterious $E_{\text {III }}$ type.

An independent analysis of invariant states has been carried out by RUELLE [6] who has obtained results similar to our theorems 2 and 3 using rather different methods. The advantage of our methods appears to be that they are open to generalisation to more complicated symmetry groups than the abelian group of space-translations.

In section 2 we begin by developing some properties of mean values of operators in Hilbert space. In section 3 we introduce the algebraic definitions which are necessary for the sequel. Section 4 is devoted to the first decomposition theorem and the characterisation of $E$-states. The second decomposition theorem and the characterisation of $L$-states is given in section 5 . We discuss possible properties of $E_{\mathrm{I}}$-state and $L$-states in section 6 and we end with a discussion of our results and their possible generalisations in Section 7.

\section{Mean values}

We start our discussion by developing some properties of means of functions and means of operator-valued functions acting on Hilbert space. In order to define the notion of mean we first introduce a special class of filters, or less generally, sequences of functions over the Euclidean space $\mathbf{T}=\mathbf{R}^{n}$ denoting points in this space by $x, y$, etc. In fact all definitions and results of this, and the two following sections can be applied to a general locally compact abelian group $\mathbf{T}$ with Haar measure $d x$ a fact which will be of relevance in section 5 .

Definition 1. An M-filter $f$ is a family of functions $f_{\alpha}$ over $\mathbf{T}$, indexed by a filter $\alpha$, which satisfies the following properties:

i) $f_{\alpha}(x) \geqq 0$

ii) $\int d x f_{\alpha}(x)=1$ for each $\alpha$,

iii) $\lim _{\alpha} \int d x\left|f_{\alpha}(x)-f_{\alpha}(x+y)\right|=0$ for all $y \in \mathbf{T}$. 
Utilising this definition we define for each $M$-filter a mean $\underline{h}_{f}$ of a complex function $h$ by

$$
\underline{h}_{f}=\lim _{\alpha} \int d x f_{\alpha}(x) h(x)
$$

whenever this definition makes sense. The mean $\underline{h}_{f}$ depends in general upon the particular choice of $M$-filter as the notation already implies. As an illustration we note that a particular choice of $f_{\alpha}$ would be the functions $V^{-1} \chi_{v}$ where $\chi_{v}$ is the characteristic function of the compact subset $v$ of volume $V$ i.e.

$$
\begin{aligned}
& \chi_{v}(x)=1 \quad \text { if } \quad x \in v \\
& =0 \quad \text { if } \quad x \notin v
\end{aligned}
$$

and with this choice the limit would be a limit $V \rightarrow \infty$ (cf. (2)).

If $x \rightarrow A(x)$ is an operator valued function acting on a Hilbert space $\mathfrak{S}$ we can define means of $A(x)$ in various ways and the most useful of these are given by the following.

Definition 2. If $x \rightarrow A(x)$ is an operator-valued function acting on a Hilbert space $\mathfrak{G}$, if $f$ is an $M$-filter and if operators ${ }_{w} \underline{A}_{f}, \underline{A}_{f}$ and ${ }_{u} \underline{A}_{f}$ on $\mathfrak{G}$ exist with the properties

$$
\begin{gathered}
\lim _{\alpha}\left(\varphi\left|\int d x f_{\alpha}(x) A(x)-{ }_{w} \underline{A}_{f}\right| \psi\right)=0 \text { for all } \varphi, \psi \in \mathfrak{G} \\
\lim _{\alpha}\left\|\left(\int d x f_{\alpha}(x) A(x)-{ }_{s} \underline{A}_{f}\right) \psi\right\|=0 \text { for all } \psi \in \mathfrak{F} \\
\lim _{\alpha}\left\|\int d x f_{\alpha}(x) A(x)-{ }_{u} \underline{A}_{f}\right\|=0
\end{gathered}
$$

respectively, then we call ${ }_{w} \underline{A}_{f}$ a weak mean, ${ }_{s} \underline{A}_{f}$ a strong mean and ${ }_{u} \underline{A}_{f} a$ uniform mean of $A(x)$.

It should be noted that these means have properties similar to those of limits, the existence of the uniform mean implies the existence of the strong mean which in turn implies the existence of the weak mean. If any two of the means of $A(x)$ exist it also follows that they are equal. We now wish to apply these definitions to some specific cases.

If $x \rightarrow U(x)$ is a strongly continuous unitary representation of the group $\mathbf{T}$ of translations in $\mathbf{R}^{n}$ by operators acting on $\mathfrak{H}$ then in general $U(x)$ has the spectral decomposition.

$$
U(x)=\sum_{n} E\left(p_{n}\right) e^{i p_{n} x}+\int d E(p) e^{i p x}
$$

where $d(\varphi|E(p)| \psi)$ is a continuous measure for all $\varphi, \psi \in \mathfrak{F}$ and $E\left(p_{n}\right)$ is the projector onto all states $(n)$ such that

$$
\left.U(x) \mid n)=e^{i p_{n} x} \mid n\right) .
$$


Lemma 1. All weak means of $U(x)$ exist and are all equal to $E(0)$. More generally, for any $p$

$$
\lim _{\alpha}\left(\varphi\left|\int d x f_{\alpha}(x) e^{-i p x} U(x)-E(p)\right| \psi\right)=0, \quad \varphi, \psi \in \mathfrak{F}
$$

where $f$ is any $M$-filter.

Proof. In order to prove (9) we first note that we can assume, without loss of generality, that

and secondly we have

$$
E(p) \mid \varphi)=0
$$

$$
\begin{aligned}
\lim _{\alpha} \mid\left(\chi\left|\left(1-e^{i p y} U(-y)\left(\int d x f_{\alpha}(x) e^{-i p x} U(x)-E(p)\right) \mid \psi\right)\right|\right. \\
=\lim _{\alpha}\left|\left(\chi\left|\int d x\left(f_{\alpha}(x)-f_{\alpha}(x+y)\right) e^{-i p x} U(x)\right| \psi\right)\right|< \\
\quad<\lim _{\alpha} \int d x\left|f_{\alpha}(x)-f_{\alpha}(x+y)\right| \\
=0
\end{aligned}
$$

for all $\chi \psi \in \mathfrak{S}$ and all $y \in \mathbf{T}$.

Now however we can, by virtue of $(10)$, choose $\chi_{i} \in \mathfrak{G}$ and $y_{i} \in \mathbf{R}^{n}$ such that for $\varepsilon>0$.

$$
\left\|\varphi-\sum_{i=1}^{n}\left(1-e^{-i p y_{i}} U\left(y_{i}\right)\right) \chi_{i}\right\|<\varepsilon .
$$

As the operator appearing in (9) is uniformly bounded with respect to $\alpha$, combining (12) and (11) gives (9).

We next deduce the existence of all strong means of $U(x)$.

Lemma 2. All strong means of $U(x)$ exist and are all equal to $E(0)$. More generally, for any $p$,

$$
\lim _{\alpha}\left\|\left(\int d x f_{\alpha}(x) e^{-i p x} U(x)-E(p)\right) \psi\right\|=0, \quad \psi \in \mathfrak{G}
$$

where $f$ is any $M$-filter.

Proof. The above argument actually establishes that

$$
\lim _{\alpha}\left\{\int d x f_{\alpha}(x) e^{-i p x} U(x)-E(p)\right\} \varphi=0
$$

for $\varphi$ in the total set

$$
E(p) \mathfrak{S} \cup\left\{\left(1-e^{-i p y} U(y)\right) \chi ; \chi \in \mathfrak{H}\right\} .
$$

Again due to uniform boundedness with respect to $\alpha$ this is enough to deduce the Lemma.

Although we do not wish to consider uniform means of $e^{-i p x} U(x)$ we mention, without proof, that such means exist if and only if the spectral measure $d E(p)$ defined by (7), vanishes in some open neighbourhood of $p$-space containing the point $p$. In this case the means are of course all equal to $E(p)$. 
The next step in our development is to use the information concerning the means of $U(x)$ to derive properties of the means of other operator-valued functions. If $A_{1}, A_{2} \ldots A_{n}$ are bounded operators acting on $\mathfrak{Y}$ we define the respective translates $A_{1}(x) \ldots A_{n}(x)$ to be the operators given by

$$
A_{i}(x)=U(x) A_{i} U(x)^{-1} \text { for } i=1,2, \ldots n .
$$

Our next result concerns means of products of operator-valued functions $A_{i}(x)$ defined in this manner.

Lemma 3. If $f^{i}$ are $n M$-filters, $i=1,2, \ldots n$, then

$$
\begin{array}{r}
\lim _{\alpha_{1}} \ldots \lim _{\alpha_{n}}\left(\chi \mid\left(\int d x_{1} \ldots d x_{n} f_{\alpha_{1}}^{1}\left(x_{1}\right) \ldots f_{\alpha_{n}}^{n}\left(x_{n}\right) A_{1}\left(x_{1}\right) \ldots A_{n}\left(x_{n}\right)-\right.\right. \\
\left.\left.-E(p) A_{1} E(p) \ldots E(p) A_{n}\right) E(p) \mid \varphi\right)=0
\end{array}
$$

and

$$
\begin{array}{r}
\lim _{\alpha_{1}} \ldots \lim _{\alpha_{n}} \|\left(\int d x_{1} \ldots d x_{n} f_{\alpha_{1}}^{1}\left(x_{1}\right) \ldots f_{\alpha_{n}}^{n}\left(x_{n}\right) A_{1}\left(x_{1}\right) \ldots A_{n}\left(x_{n}\right)-\right. \\
\left.-E(p) A_{1} E(p) \ldots E(p) A_{n}\right) E(p) \psi \|=0
\end{array}
$$

for all $\chi, \varphi, \psi \in \mathfrak{Y}$, for all $p$, and for any order or manner of the limits i.e. the limit may be taken with respect to the product filter.

Proof. We note that.

$$
\begin{gathered}
\mid\left(\chi \mid\left(\int d x_{1} \ldots d x_{n} f_{\alpha_{1}}^{1}\left(x_{1}\right) \ldots f_{\alpha_{n}}^{n}\left(x_{n}\right) A_{1}\left(x_{1}\right) \ldots A_{n}\left(x_{n}\right)-\right.\right. \\
\left.\left.\quad-E(p) A_{1} E(p) \ldots A_{n}\right) E(p) \mid \varphi\right) \mid \\
=\mid \sum_{r=1}^{n}\left(\chi \mid \int d x_{1} \ldots d x_{r} f_{\alpha_{1}}^{1}\left(x_{1}\right) \ldots f_{\alpha_{r}}^{r}\left(x_{r}\right) A_{1}\left(x_{1}\right) \ldots\right. \\
\left.\ldots A_{r}\left(x_{r}\right) E(p) A_{r+1} \ldots A_{n} E(p) \mid \varphi\right)- \\
-\left(\chi \mid \int d x_{1} \ldots d x_{r} f_{\alpha_{1}}^{1}\left(x_{1}\right) \ldots f_{\alpha_{r-1}}^{r-1}\left(x_{r-1}\right) A_{1}\left(x_{1}\right) \ldots\right. \\
\left.\ldots A_{r-1}\left(x_{r-1}\right) E(p) A_{r} \ldots A_{n} E(p) \mid \varphi\right) \mid \\
=\mid \sum_{r=1}^{n}\left(\chi \mid \int d x_{1} \ldots d x_{r} f_{1}^{\prime}\left(x_{1}\right) \ldots f_{\alpha_{r}}^{r}\left(x_{r}\right) A_{1}\left(x_{1}\right) \ldots\right. \\
\left.\ldots A_{r-1}\left(x_{r-1}\right)\left(e^{-i p x} U(x)-E(p)\right) \cdot A_{r} E(p) \ldots A_{n} E(p) \mid \varphi\right) \mid \leqq \\
\leqq \sum_{r=1}^{n}\|\chi\| \cdot\left\|A_{1}\right\| \ldots\left\|A_{r-1}\right\| \| \int d x_{r} f_{\alpha_{r}}^{r}\left(x_{r}\right)\left(e^{-i p x} U(x)-E(p)\right) \times \\
\left.\times A_{r} E(p) \ldots A_{n} E(p) \mid \varphi\right) \| .
\end{gathered}
$$

Now combining inequality (19) and (13) we can deduce (17). In an analogous manner we may deduce (18).

Finally we note that even if the spectrum of $U(x)$ is purely continuous lemmas 1 and 2 provide non-trivial results but lemma 3 is an empty statement. In this case it is therefore necessary to develop more 
powerful methods for investigating means of the translates $A_{i}(x)$. In the sequel we will apply the above results to certain algebras of operators and we prepare the ground for these applications by introducing the required algebraic definitions and notations in the next section.

\section{Algebraic definitions}

In the following we are interested in examining a $C^{*}$-algebra $\mathfrak{A}$ on which the group $\mathbf{T}$ of space-translations acts as a group of automorphisms (for general information on $C^{*}$-algebras see [7] or, for a short resumé, the appendix of [8]). We use the notation $A \in \mathfrak{A} \rightarrow A(x) \in \mathfrak{A}$ to describe the action of these automorphisms and assume strong continuity i.e. we assume

$$
\|A(x)-A\| \underset{|x|=0}{\longrightarrow} 0
$$

where\| $\|$ denotes the algebraic norm. We will limit our discussion to the consideration of invariant states over $\mathfrak{A}$. Explicitly a state $\Phi$ over $\mathfrak{A}$ is a positive linear form over $\mathfrak{A}$ and an invariant state is specified by the additional property

$$
\Phi(A(x))=\Phi(A)
$$

for all $x \in \mathbf{R}^{n}$ and $A \in \mathfrak{A}$. It is well known that from each state one can construct a cyclic representation of $\mathfrak{A}$ by operators $\pi(\mathfrak{A})$ acting in a Hilbert space $\mathfrak{S}$ and that from an invariant state it is also possible to construct a unitary representation $U(x)$ of the group $\mathbf{T}$ for which the cyclic vector $\Omega$ is invariant. The connection between these quantities is given by

$$
\begin{aligned}
\text { i) } \quad \Phi(A) & =(\Omega|\pi(A)| \Omega), \\
\text { ii) } \pi(A(x)) & =U(x) \pi(A) U(x)^{-1}, \\
\text { iii) } \quad U(x) \Omega & =\Omega .
\end{aligned}
$$

Due to property ii) we call $\pi$ a $\mathbf{T}$-covariant representation of $\mathfrak{A}$. We have previously shown in DKR that all T-covariant representations of $\mathfrak{A}$, of which the above are a special subclass, can be best studied by considering an algebra $\mathfrak{A}_{1}^{T}$ which is constructed from $\mathfrak{A}$ and $\mathbf{T}$ in such a manner that its *-representations $\hat{\pi}$ are in one-to-one correspondence with the T-covariant representations $(\pi, U)$ of $(\mathfrak{A}, \mathbf{T})$. The algebra $\mathfrak{A}_{1}^{T}$, which we call a covariance algebra, is of use in the ensuing analysis and we refer to DKR for details of its construction etc.; for our present purposes it suffices to note the following facts. If $\hat{\pi}$ is a representation of $\mathfrak{Q}_{1}^{T}$ then it is connected to representations $\pi$ and $U$, of $\mathscr{A}$ and $T$ respectively, by

$$
\hat{\pi}(X)=\int d u \pi\left(X_{u}\right) U(u)
$$


where $X \in \mathfrak{A}_{1}^{T}$ is an $\mathbf{I}_{1}$-integrable function from the space $\mathbf{T}$ to $\mathfrak{A}$ i.e. where $u \in \mathbf{T} \rightarrow X_{u} \in \mathfrak{Z}$ is such that

$$
\int d u\left\|X_{u}\right\|<\infty .
$$

Further, it is possible to extend the T-automorphism of $\mathfrak{A}$ to automorphisms of $\mathfrak{A}_{1}^{T}$ by defining for each $X \in \mathfrak{U}_{1}^{T}$ the translate $X(x)$ through

$$
\{X(x)\}_{u}=X_{u}(x)
$$

and thus in the representation $\hat{\pi}$ we have

$$
\hat{\pi}(X(x))=U(x) \hat{\pi}(X) U(x)^{-1}=\int d u \pi\left(X_{u}\right) U(u) .
$$

Finally it is possible to extend an invariant state $\Phi$ over $\mathfrak{A}$ to an invariant state $\hat{\Phi}$ over $\mathfrak{U}_{1}^{T}$ through the definition

$$
\hat{\Phi}(X)=\int d u \Phi\left(X_{u}\right)
$$

and the representations $(\pi, U)$ and $\hat{\pi}$ associated with $\Phi$ and $\hat{\Phi}$ respectively act in the same Hilbert space $\mathfrak{G}$. The invariant vector $\Omega$ is cyclic for both $\pi(\mathfrak{A})$ and $\hat{\pi}\left(\mathfrak{A}_{1}^{T}\right)$. We will call $\hat{\Phi}$ the canonical extension of $\Phi$.

In the following section we will also need the concept of an enveloping von Neumann algebra (see [7] Chapter 12) as applied specifically to a covariance algebra $\mathfrak{A}_{1}^{T}$. The enveloping von Neumann algebra $\mathfrak{A}_{1}^{T * *}$ of $\mathscr{U}_{1}^{T}$ is the dual of the Banach space $\mathfrak{A}_{1}^{T *}$, which consists of the continuous linear forms on $\mathfrak{A}_{1}^{T}$ i.e. $\mathfrak{A}_{1}^{T * *}$ is the bi-dual of $\mathfrak{A}_{1}^{T}$.

An alternative definition is given by identifying $\mathfrak{A}_{1}^{T * *}$ with the weak closure of the set of representatives of $\mathfrak{A}_{1}^{T}$ contained in the "universal representations" $\hat{\tau}$ which is in turn defined to be the direct sum of all cyclic representations of $\mathfrak{A}_{1}^{T}$. From this latter definition it is clear that $\mathfrak{Q}_{1}^{T * *}$ is a von Neumann algebra containing $\mathfrak{A}_{1}^{T}$ in the sense that it contains the set $\hat{\tau}\left(\mathfrak{A}_{1}^{T}\right)$ which is in one-to-one correspondence with $\mathfrak{A}_{1}^{T}$ because $\hat{\tau}$ is a faithful representation. As a general feature of the theory of von Neumann enveloping algebras an arbitrary representation $\hat{\pi}$ of $\mathfrak{A}_{1}^{T}$ (a state $\hat{\Phi}$ over $\mathfrak{2}_{1}^{T}$ ) can be uniquely extended to a $W^{*}$-representation of $\mathfrak{A}_{1}^{T * *}$ (a weakly continuous state over $\mathfrak{A}_{1}^{T * *}$ ). Due to the definition of $\mathscr{A}_{1}^{T}$ the representation $\hat{\tau}$ determines representations $\left(\tau, U_{\tau}\right)$ of $(\mathfrak{A}, \mathbf{T})$ and we note (DKR end of Theorem 3$)$ that both $\tau(\mathfrak{A})$ and $U_{\tau}(\mathbf{T})$ are contained in $\mathfrak{A}_{1}^{T * *}$. Furthermore, $\tau(\mathfrak{A})$ and $U_{\tau}(\mathbf{T})$ are faithful representations of $\mathfrak{A}$ and $\mathbf{T}$ as a consequence of $\hat{\tau}$ being faithful and thus $\mathfrak{U}$ and $\mathbf{T}$ are embedded in $\mathfrak{2}_{1}^{T * *}$ as a sub-algebra and a group of unitaries respectively. We can also consider the group algebra $\mathbf{L}_{1}(\mathbf{T})$ of $\mathbf{T}$ as a sub-*algebra of $\mathfrak{A}_{1}^{T * *}$ by assigning to every $f \in \mathbf{I}_{1}(\mathbf{T})$ the operator $\hat{\tau}(f)$ where

$$
\hat{\tau}(f)=\int d x f(x) U_{\tau}(x) .
$$

The embedding follows because the definition $f \rightarrow \hat{\tau}(f)$ is easily seen to provide a faithful representation of $\mathbf{L}_{1}(\mathbf{T})$ such that $\hat{\tau}(f)$ commutes with 
the commutant of $U_{\tau}(\mathbf{T})$. Finally all the spectral projectors of the representation $U_{\tau}(\mathbf{T})$ will be in the weak closure of the sub-algebra $\mathbf{L}_{1}(\mathbf{T})$ of $\mathfrak{A}_{1}^{T * *}$ and in particular the associated projector $E_{\tau}(0)$ onto the invariant states is an element of $\mathfrak{A}_{1}^{T * *}$. This last fact will be of importance in the sequel.

Next we recall that a state $\Phi$ over $\mathfrak{A}$ is said to be strongly clustering if

$$
|\Phi(A(x) B)-\Phi(A(x)) \Phi(B)| \underset{|x|=\infty}{\longrightarrow} 0 \text { for all } A, B \in \mathfrak{A}
$$

and we will call a state weakly clustering (cf. DKR, section VI) if

$$
\lim _{\alpha} \int d x f_{\alpha}(x)\{\Phi(A(x) B)-\Phi(A(x)) \Phi(B)\}=0
$$

for all $A, B \in \mathfrak{A}$ and for some $M$-filter $f$. These definitions are principally motivated by physical considerations. If, for example $A(x)$ represents a measurement at the point $x$ and $\Phi(A(x))$ denotes the value obtained by making this measurement in the state $\Phi$ then (28) implies that the result of the product measurement $A(x) B$ is equal to the product of the results of the individual measurements in the limit $|x| \rightarrow \infty$. This interpretation implies the absence of infinite range correlations in the state $\Phi$. Alternatively the weak clustering definition (29) implies that any long range correlations in the state $\Phi$ have at most an oscillatory nature. However the latter definition also has a direct mathematical motivation which is provided by

Theorem 1. Let $\mathfrak{A}$ be a $C^{*}$-algebra on which the group $\mathbf{T}$ acts as a group of automorphisms, $\Phi$ be an invariant state over $\mathfrak{A}$ and $\hat{\Phi}$ the canonical extension of $\Phi$ to $\mathfrak{A}_{1}^{T}$. If $(\pi, U)$ and $\hat{\pi}$ are the representations of $(\mathfrak{A}, \mathbf{T})$ and $\mathfrak{A}_{1}^{T}$ on the Hilbert space $\mathfrak{S}$ and $\Omega$ the invariant vector, in $\mathfrak{H}$, determined by $\Phi$, or $\hat{\Phi}$, then the following are equivalent:

i) $\Phi$ is a weakly clustering state

ii) $\Omega$ is the only invariant vector in $\mathfrak{H}$

iii) $\lim _{\alpha} \int d x f_{\alpha}(x)\left(\psi\left|U(x)-E_{\Omega}\right| \varphi\right)=0, \psi, \varphi \in \mathfrak{G}$

iv) $\lim _{\alpha}\left\|\int d x f_{\alpha}(x)\left(U(x)-E_{\Omega}\right) \psi\right\|=0, \psi \in \mathfrak{H}$

where $E_{\Omega}$ is the projector on $\Omega$, and $f$ is any $M$-filter (thus (29) is valid for all $M$-filters).

Further, it follows from each of the equivalent conditions i)-iv) that $(\pi, U)$ is irreducible i.e. $\hat{\pi}$ is irreducible or, equivalently, $\hat{\Phi}$ is a pure state over $\mathfrak{A}_{1}^{T}$.

Proof. It is clear that iv) implies iii) but the proof of lemma 2 shows that the converse is also true. Further Lemma 1 shows that ii) and iii) are equivalent. If we next rewrite the definition of weak clustering, with the aid of (22), in the form

$$
\lim _{\alpha} \int d x f_{\alpha}(x)\left\{\left(\Omega\left|\pi(A)\left(U(x)^{-1}-E_{\Omega}\right) \pi(B)\right| \Omega\right)\right\}=0
$$


we immediately see that iii) implies i). However as $\Omega$ is cyclic for $\pi$ we also deduce from (30) that i) implies iii). This demonstrates the equivalence of statements i) -iv). Finally we remark that (30) establishes that $E_{\Omega}$ is in the weak closure of $(\pi, U)$ but as $\Omega$ is cyclic for $\pi$ it must follow that $(\pi, U)$ is irreducible.

Up to this stage we have considered quite general $C^{*}$-algebras acted on by the group of automorphisms $\mathbf{T}$ and we have placed no structure requirements upon these algebras. In this broad framework theorem 1 appears to be an optimal result. However, in physics, one is interested in algebras with properties reflecting local structure and therefore in the following we will restrict our attention to a special class of algebras which are characterised by an 'asymptotically local' property. These algebras, which were first introduced in DKR under the name of asymptotically abelian algebras, are, as discussed in the introduction, of special interest in Statistical Mechanics.

Definition 3. An asymptotically abelian algebra $\mathfrak{A}$ is a $C^{*}$-algebra acted on by the group of automorphism $\mathbf{T}$ with the property

for all $A, B \in \mathfrak{A}$.

$$
\|[A(x), B]\| \underset{|x|=\infty}{\longrightarrow} 0
$$

In the ensuing sections we will analyse the structure of invariant states over such algebras and will see that the result of theorem 1 can be considerably strengthened in this case (cf. theorem 3).

\section{Invariant States}

The major aims of this section are, firstly, to show that any invariant state over an asymptotically abelian algebra can be uniquely decomposed into invariant weakly clustering states, secondly, to characterise and, thirdly, to classify these latter states. As a preliminary, and also an aid, to this investigation we first derive the two following lemmas.

Lemma 4. If $\Phi$ is an invariant state over an asymptotically abelian algebra and $(\pi, U)$ are the representations of $(\mathcal{U}, \mathbf{T})$ associated with $\Phi$ then, for any $p_{l}, p_{n}, p_{m}$ and all $A, B \in \mathfrak{A}$,

$$
\begin{aligned}
E\left(p_{l}\right) \pi(A) E\left(p_{l}+p_{m}\right) \pi(B) & E\left(p_{n}\right) \\
& =E\left(p_{l}\right) \pi(B) E\left(p_{n}-p_{m}\right) \pi(A) E\left(p_{n}\right)
\end{aligned}
$$

where the $E\left(p_{n}\right)$ are the projectors onto discrete eigenstates of $U(x)$ defined by the spectral decomposition (7). In particular for any $p_{n}$ and all $A, B \in 2$

$$
\left[E\left(p_{n}\right) \pi(A) E\left(p_{n}\right), E\left(p_{n}\right) \pi(B) E\left(p_{n}\right)\right]=0 .
$$

Proof. As $\mathfrak{A}$ is an asymptotically abelian algebra we may deduce from (31) that

$$
e^{i p_{m} x}\left(\psi\left|E\left(p_{1}\right)[\pi(A(x)), \pi(B)] E\left(p_{n}\right)\right| \varphi\right) \underset{|x|=\infty}{\longrightarrow} 0
$$


for any $p_{l}, p_{m}, p_{n}$, all $A, B \in \mathfrak{U}$ and for all vectors $\psi, \varphi$ in the representation space $\mathcal{S}$. However (34) implies that

$$
\lim _{\alpha} \int d x f_{\alpha}(x) e^{i p_{m} x}\left(\psi\left|E\left(p_{l}\right)[\pi(A(x)), \pi(B)] E\left(p_{n}\right)\right| \varphi\right)=0
$$

for any $M$-filter $f$. Next, using the T-covariance property

and the fact that

$$
\pi(A(x))=U(x) \pi(A) U(x)^{-1}
$$

$$
U(x) E\left(p_{n}\right)=e^{i p_{n} x} E\left(p_{n}\right)
$$

we can rewrite (35) in the form

$$
\begin{aligned}
& \lim _{\alpha} \int d x f_{\alpha}(x)\left(\psi \mid E\left(p_{l}\right)\left\{\pi(A) e^{i\left(p_{l}+p_{m}\right) x} U(x)^{-1} \pi(B)-\right.\right. \\
&\left.\left.-\pi(B) e^{-i\left(p_{n}-p_{m}\right) x} U(x) \pi(A)\right\} E\left(p_{n}\right) \mid \varphi\right)=0 .
\end{aligned}
$$

Application of the result (9) of Lemma 1 to (38) delivers (32). In order to deduce (33) from (32) it is only necessary to choose $p_{l}=p_{n}$ and $p_{m}=0$.

Lemma 5. If $\Phi$ is an invariant state over an asymptotically abelian algebra $\mathfrak{A}$ and $(\pi, U)$ are the representations of $(\mathfrak{A}, \mathfrak{T})$ associated with $\Phi$ then all strong means of $\pi(A(x))$ exist, are independent of the choice of $M$-filter $f$, are in the centre of the von Neumann algebra generated by $(\pi, U)$ and, denoting the strong means by ${ }_{s} \pi(A)$ we have

$$
{ }_{s} \pi(A) \pi(B) \Omega=\pi(B)(E(0) \pi(A) E(0)) \Omega .
$$

Thus if $\hat{\Phi}$ is the canonical extension of $\Phi$ to $\mathfrak{A}_{1}^{T}$ and $\hat{\pi}$ is the associated representation of $\mathfrak{A}_{1}^{T}$ then ${ }_{s} \pi(A)$ is in the centre $\mathcal{B}_{\hat{\imath}}$ of the von Neumann algebra generated by $\hat{\pi}$. Further $\mathcal{B}_{\hat{\imath}}$ is the smallest von Neumann algebra containing all strong means ${ }_{s} \pi(A)$ and the mapping $Z \in \mathcal{B}_{\hat{\pi}} \rightarrow E(0) Z E(0)$ defines an isomorphism between von Neumann algebras. Moreover $\mathcal{B}_{\hat{\imath}}$ $=\hat{\pi}\left(\mathfrak{Q}_{1}^{T}\right)^{\prime}$.

Proof. Recalling the definition (6) of strong means of operator-valued functions we see that the existence of ${ }_{s} \pi(A)$ its independence of the choice of $M$-filter $f$, and its action (39), are all established if we can prove that

$$
\lim _{\alpha}\left\|\int d x f_{\alpha}(x) \pi(A(x)) \pi(B) \Omega-\pi(B)(E(0) \pi(A) E(0)) \Omega\right\|=0
$$

because $\{\pi(B) \Omega ; B \in \mathfrak{A}\}$ is a dense set of vectors and

However

$$
\left\|\int d x f_{\alpha}(x) \pi(A(x))\right\| \leqq\|A\| .
$$

$$
\begin{aligned}
&\left\|\int d x f_{\alpha}(x)(\pi(A(x)) \pi(B)-\pi(B) E(0) \pi(A) E(0)) \Omega\right\| \leqq \\
& \leqq\left\|\int d x f_{\alpha}(x)[\pi(A(x)), \pi(B)] \Omega\right\|+ \\
&+\left\|\pi(B) \int d x f_{\alpha}(x)(\pi(A(x))-E(0) \pi(A)) \Omega\right\| \leqq \\
& \leqq \int d x f_{\alpha}(x)\|[\pi(A(x)), \pi(B)]\|+ \\
&+\|\pi(B)\|\left\|\int d x f_{\alpha}(x)(U(x)-E(0)) \pi(A) \Omega\right\|
\end{aligned}
$$


and in the filter limit the first term of (41) tends to zero due to (31) and the second term due to Lemma 2. Now that we have established that ${ }_{s} \pi(A)$ exists and acts in the manner described by (39) it is easy to demonstrate that it is in the centre of the von Neumann algebra generated by $(\pi, U)$. In particular we have by definition that ${ }_{s} \pi(A)$ is in the weak closure of $\pi$ so it is only necessary to show that it commutes with $(\pi, U)$. This latter fact is, however, a consequence of (39) e.g.

$$
\begin{aligned}
{ }_{s} \pi(A) \pi(C) \pi(B) \Omega & =\pi(C) \pi(B) E(0) \pi(A) E(0) \Omega \\
& =\pi(C){ }_{s} \pi(A) \pi(B) \Omega .
\end{aligned}
$$

The second statement of the Lemma follows from the fact that the von Neumann algebras generated by $(\pi, U)$ and $\hat{\pi}$ are identical (DKR end of the theorem 3). We note that the abelian character of the set $\left\{{ }_{s} \pi(A) ; A \in \mathfrak{A}\right\}$ is a consequence of (39) and (33). The final statement of the Lemma is established by noting that $Z \in B_{\hat{\imath}}$ has the property $[Z, \pi(B)]=0=[Z, E(0)]$, hence

and therefore

$$
Z_{\pi}(B) \Omega=\pi(B) E(0) Z E(0) \Omega
$$

$$
\left(Z-{ }_{s} \pi(A)\right) \pi(B) \Omega=\pi(B) E(0)(Z-\pi(A)) E(0) \Omega
$$

from which the stated facts immediately follow.

Finally we deduce that $\mathcal{B}_{\hat{\imath}}=\hat{\pi}\left(\mathfrak{Q}_{1}^{T}\right)^{\prime}$ by showing that this commutant is abelian. Now $\hat{\pi}\left(\mathfrak{U}_{1}^{T}\right)^{\prime}$ is isomorphic to $E(0) \hat{\pi}\left(\mathfrak{U}_{1}^{T}\right)^{\prime} E(0)$ because $E(0) \in \hat{\pi}\left(\mathfrak{Q}_{1}^{T}\right)^{\prime \prime}$ and $\Omega \in E(0) \mathfrak{S}$ is cyclic. But

$E(0) \hat{\pi}\left(\mathfrak{U}_{1}^{T}\right)^{\prime} E(0) \leqq E(0)\left(E(0) \hat{\pi}\left(\mathfrak{A}_{1}^{T}\right) E(0)\right)^{\prime} E(0)=\left(E(0) \hat{\pi}\left(\mathfrak{U}_{1}^{T}\right) E(0)\right)^{\prime \prime}$

where the last equality follows because $\Omega$ is cyclic, in $E(0) \mathfrak{H}$, for the abelian algebra $E(0) \hat{\pi}\left(\mathfrak{U}_{1}^{T}\right) E(0)$ (see [10] page 89). Hence $\hat{\pi}\left(\mathfrak{U}_{1}^{T}\right)^{\prime}$ is isomorphic to an abelian algebra and is thus abelian. (Quite generally this is the case for any von Neumann algebra whose commutant contains an abelian projector with a cyclic vector in its range.)

We are now in a position to derive the main decomposition theorem for invariant states.

Theorem 2. If $\Phi$ is an invariant state over an asymptotically abelian algebra $\mathfrak{A}$ and the associated representation $\pi$ acts in a separable Hilbert space $\mathfrak{S}$ then there exists a unique decomposition of $\Phi$

$$
\Phi=\int d \mu(\lambda) \Phi_{\lambda}
$$

into invariant weakly clustering states $\Phi_{\lambda}$ over $\mathfrak{A}$ i.e. for each $A \in \mathfrak{A}$ one has the Radon integral

$$
\Phi(A)=\int d \mu(\lambda) \Phi_{\lambda}(A),
$$

and the states $\Phi_{\lambda}$ generate representations $\pi_{\lambda}$ which are mutually inequivalent. 
Further, if $\Phi$ and $\Phi_{\lambda}$ are the canonical extensions of $\Phi$ and $\Phi_{\lambda}$ to $\mathfrak{A}_{1}^{T}$, respectively, then the corresponding decomposition

$$
\hat{\Phi}(X)=\int d \mu(\lambda) \hat{\Phi}_{\lambda}(X), \quad X \in \mathfrak{A}_{1}^{T}
$$

is the central decomposition of $\hat{\Phi}$ over $\mathfrak{A}_{1}^{T}$ and this decomposition is into pure states.

Corresponding to the above decompositions we have the associated decompositions of the representations e.g.

$$
\hat{\pi}(X)=\int^{\oplus} d \mu(\lambda) \hat{\pi}_{\lambda}(X), \quad X \in \mathfrak{A}_{1}^{T} .
$$

Proof. The existence of the decomposition (43) will be deduced from the existence of the decomposition (46). Since $\hat{\pi}$ acts on a separable Hilbert space $\mathfrak{S}$ it has a unique central decomposition which, according to Lemma 5 , is a decomposition into irreducibles.

Specifically $\mathfrak{G}$ is the direct ingegral of Hilbert spaces $\mathfrak{G}_{\lambda}$

$$
\mathfrak{F}=\stackrel{\oplus}{\int} d \mu(\lambda) \mathfrak{H}_{\lambda}
$$

and the operators $B \in \hat{\pi}\left(\mathfrak{A}_{1}^{T}\right)^{\prime \prime}$ are the decomposable operators

$$
B=\int^{\oplus} d \mu(\lambda) B_{\lambda} \quad B \in \hat{\pi}\left(\mathscr{R}_{1}^{T}\right)^{\prime \prime}
$$

whilst the operators $T$ of the center $\mathcal{B}_{\hat{\imath}}=\hat{\pi}\left(\mathfrak{A}_{1}^{T}\right)^{\prime}$ are the diagonalizable operators

$$
T=\int^{\oplus} d \mu(\lambda) T(\lambda), \quad T(\lambda) \in \mathbf{C}, T \in \mathcal{B}_{\hat{\imath}} .
$$

If we consider (47) for $B=\hat{\pi}(X), X \in \mathscr{Q}_{1}^{T}$ we obtain the central decomposition of $\hat{\pi}$ into irreducible representation $\hat{\pi}_{\lambda}$ of $\mathfrak{A}_{1}^{T}$ :

$$
\hat{\pi}(X)=\int^{\oplus} d \mu(\lambda) \hat{\pi}_{\lambda}(X) \quad X \in \mathfrak{A}_{1}^{T}
$$

where $\hat{\pi}_{\lambda}(X)$ is defined to be equal to $\{\hat{\pi}(X)\}_{\lambda}$.

On the other hand the cyclic vector $\Omega \in \mathfrak{F}$ associated to the positive form $\hat{\Phi}$ is decomposed as

$$
\Omega=\int^{\oplus} d \mu(\lambda) \Omega_{\lambda} \quad \Omega_{\lambda} \in \mathfrak{G}_{\lambda}
$$

where $\Omega_{\lambda} \neq 0$ for almost all $\lambda$ (see [7], 8.8.1). Hence

$$
\hat{\Phi}(X)=\int^{\oplus} d \mu(\lambda) \hat{\Phi}_{\lambda}(X)
$$

where $\Phi_{\lambda}$ is defined by

$$
\hat{\Phi}_{\lambda}(X)=\left(\Omega_{\lambda}\left|\hat{\pi}_{\lambda}(X)\right| \Omega_{\lambda}\right)_{\mathfrak{S} \lambda} \quad X \in \mathfrak{A}_{1}^{T} .
$$

Now let $(\pi, U)$ and $\left(\pi_{\lambda}, U_{\lambda}\right)$ be the covariant representations of $(\mathfrak{A}, \mathbf{T})$ corresponding to $\hat{\pi}$ and $\hat{\pi}_{\lambda}$ respectively. We have for $A \in \mathfrak{A}, x \in \mathbf{T}$ and 
$X \in \mathfrak{A}_{1}^{T}$, (see $[1]$ theorem 3 )

$$
\begin{aligned}
& \pi(A) \hat{\pi}(X)=\pi(\varrho(A) X)=\int d \mu(\lambda) \hat{\pi}_{\lambda}(\varrho(A) X)=\int d \mu(\lambda) \pi_{\lambda}(A) \hat{\pi}_{\lambda}(X) \\
& U(x) \hat{\pi}(X)=\pi(V(x) X)=\int d \mu(\lambda) \hat{\pi}_{\lambda}(V(x) X)=\int d \mu(\lambda) U_{\lambda}(x) \hat{\pi}_{\lambda}(X)
\end{aligned}
$$

from which, using cyclicity of the vector (49), one deduces

Furthermore, for all $x \in \mathbf{T}$

$$
\begin{aligned}
& \pi(A)=\int^{\oplus} d \mu(\lambda) \pi_{\lambda}(A) \\
& U(x)=\int^{\oplus} d \mu(\lambda) U_{\lambda}(x) .
\end{aligned}
$$

$$
U(x) \Omega=\int^{\oplus} d \mu(\lambda) U_{\lambda}(x) \Omega_{\lambda}=\Omega=\int^{\oplus} \Omega_{\lambda} d \mu(\lambda)
$$

whence, for almost all $\lambda$

$$
U_{\lambda}(x) \Omega_{\lambda}=\Omega_{\lambda} \text { for all } x \in \mathbf{T}
$$

(this is first proven for all rational $x$ and follows for all $x$ by the strong continuity of $\left.U_{\lambda}(x)\right)$. This shows that the pure state $\hat{\Phi}_{\lambda}$ is an invariant state. The decomposition for the state $\Phi$ over $\mathfrak{A}$ resulting from (52a) and (49):

$$
\Phi(A)=(\Omega|\pi(A)| \Omega)=\int d \mu(\lambda) \Phi_{\lambda}(A) \quad A \in \mathfrak{A}
$$

where $\Phi_{\lambda}$ is defined by

$$
\Phi_{\lambda}(A)=\left(\Omega_{\lambda}\left|\pi_{\lambda}(A)\right| \Omega_{\lambda}\right)_{\mathfrak{S} \lambda}
$$

is therefore a decomposition into invariant states generating covariant representations $\left(\pi_{\lambda}, U_{\lambda}\right)$ such that the set $\pi_{\lambda}(\mathfrak{A}) \cup U(\mathbf{T})$ is irreducible. We have shown in [1] theorem 7 (cf. theorem 3 below) that this property is equivalent to weak clustering for those states $\Phi_{\lambda}$.

To demonstrate the inequivalence of the $\pi_{\lambda}$ generated by the $\Phi_{\lambda}$ we first deduce from (32) that

$$
E\left(p_{l}\right) \pi(A) E\left(p_{l}\right) \pi(B) E\left(p_{n}\right)=E\left(p_{l}\right) \pi(B) E\left(p_{n}\right) \pi(A) E\left(p_{n}\right) .
$$

However, if $(\pi, U)$ is a covariant representation associated with a weakly clustering state $\Phi$ we will prove in Theorem 4 that the projectors $E\left(p_{l}\right), E\left(p_{n}\right)$, onto discrete eigenstates $\left.\left.\mid l\right), \mid n\right)$ of $U(x)$, are at most one dimensional. Thus we have

$$
(l|\pi(A)| l)=(n|\pi(A)| n)
$$

and hence $\Phi$ is the only invariant state in the representation it generates. Thus if $\Phi_{\lambda_{1}} \Phi_{\lambda_{2}}$ generate equivalent representations then $\Phi_{\lambda_{1}}=\Phi_{\lambda_{2}}$ which is contrary to the construction for $\lambda_{1} \neq \lambda_{2}$.

The general idea of the uniqueness proof is to reduce a given decomposition into invariant weakly clustering states to a decomposition into pure states over an abelian $C^{*}$-algebra. In this latter case the decomposition is known to be unique. The abelian $C^{*}$-algebra which we 
consider is the smallest norm-closed sub*-algebra of the enveloping von Neumann algebra $\mathfrak{U}_{1}^{T * *}$ containing all operators of the form $E_{\tau}(0) \tau(\mathfrak{A}) E_{\tau}(0)$. We will denote this algebra by $\mathfrak{E}$. It is abelian as a consequence of (33) of Lemma 4.

Let us first define a linear one-to-one mapping of the invariant states $\Phi$ over $\mathfrak{A}$ to states $\phi$ over $\mathfrak{\xi}$. This mapping is obtained by first extending $\Phi$ over $\mathfrak{A}$ to a $\hat{\Phi}$ over $\mathfrak{A}_{1}^{T}$ by the canonical procedure (27), then extending $\Phi$ to a weakly continuous state $\bar{\Phi}$ over $\mathfrak{Q}_{1}^{T * *}$ and finally taking the restriction $\phi$ of $\bar{\Phi}$ to the subalgebra $\mathbb{E}$. This procedure is well defined because each step is uniquely specified, it is obviously linear and it remains to be shown that it is one-to-one. To demonstrate this property we first recall that $\hat{\Phi}$ is an invariant state over $\mathfrak{A}_{1}^{w}$ and as an immediate consequence $\bar{\Phi}$ is such that

$$
\bar{\Phi}\left(U_{\tau}(x) X U_{\tau}(-x)\right)=\bar{\Phi}(X) \quad X \in \mathcal{A}_{1}^{T * *}
$$

thus $\phi$ will have the property

$$
\begin{aligned}
\phi\left(E_{\tau}(0) \tau(A) E_{\tau}(0)\right) & =\bar{\Phi}\left(E_{\tau}(0) \tau(A) E_{\tau}(0)\right) \\
& =\bar{\Phi}(\tau(A))=\Phi(A) .
\end{aligned}
$$

Therefore $\phi$ determines $\Phi$ and the mapping is one-to-one.

Next we demonstrate that the weak clustering property of $\Phi$ over $\mathfrak{U}$ is equivalent to the purity of $\phi$ over $\mathfrak{F}$. This is established by noting that

$$
\begin{aligned}
\Phi(A(x) B)-\Phi(A) \Phi(B) & =\phi\left(E_{\tau}(0) \tau(A) U_{\tau}(-x) \tau(B) E_{\tau}(0)\right)- \\
& -\phi\left(E_{\tau}(0) \tau(A) E_{\tau}(0)\right) \phi\left(E_{\tau}(0) \tau(B) E_{\tau}(0)\right)
\end{aligned}
$$

and taking means of both sides and using Lemma 1 we see that the weak clustering of $\Phi$ is equivalent to the property

$$
\begin{aligned}
\phi\left(E_{\tau}(0) \tau(A) E_{\tau}(0) \tau(B)\right. & \left.E_{\tau}(0)\right) \\
& =\phi\left(E_{\tau}(0) \tau(A) E_{\tau}(0)\right) \phi\left(E_{\tau}(0) \tau(B) E_{\tau}(0)\right)
\end{aligned}
$$

for $\phi$. But this last condition amounts to multiplicativity of $\phi$ over $\mathbb{E}$ or, since $\mathbb{E}$ is abelian, purity of $\phi$.

Now let us assume that we are given a decomposition of $\Phi$ into weakly clustering states

$$
\Phi(A)=\int d \nu(\xi) \Phi_{\xi}(A) \quad A \in \mathfrak{A}
$$

where the integral is a Radon integral taken over some locally compact space. We will prove that this decomposition induces a corresponding decomposition

$$
\phi(M)=\int d \nu(\xi) \phi_{\xi}(M) \quad M \in \mathbb{E}
$$

where the $\phi$ and $\phi_{\xi}$ are obtained from the $\Phi$ and $\Phi_{\xi}$ as explained above. 
Thus the $\phi_{\xi}$ will be pure states over $\mathbb{E}$ and the uniqueness of decomposition into pure states for an abelian $C^{*}$-algebra, together with the fact that the correspondence $\Phi \rightarrow \phi$ is one-to-one will entail uniqueness of the decomposition (58).

The passage from (58) to (59) is effected in the following manner. We first note that due to the definition (27) of the canonical extension we can deduce from (58) the decomposition

or identically,

$$
\hat{\Phi}(X)=\int d v(\xi) \hat{\Phi}_{\xi}(X), \quad X \in \mathfrak{A}_{1}^{T}
$$

$$
\bar{\Phi}(X)=\int d \nu(\xi) \bar{\Phi}_{\xi}(X), \quad X \in \mathfrak{A}_{1}^{T}
$$

where we have used Fubini's theorem to interchange the order of integrations. Now to complete the deduction of (59) we establish that (61) is valid not only for $X \in \mathfrak{A}_{1}^{T}$ but also for $M \in \mathbb{E}$. To demonstrate this, we show that an $M \in \mathbb{E}$ can be approximated in the weak topology of $\mathfrak{Q}_{1}^{T^{* *}}$ by a sequence of elements of $\mathfrak{A}_{1}^{T}$ and then we can use Lebesgue's dominated convergence theorem to interchange the integration in (61) with the weak limit. As $\mathbb{F}$ is the norm closure of linear combinations of the form

$$
E_{\tau}(0) \tau\left(A_{1}\right) E_{\tau}(0) \tau\left(A_{2}\right) \ldots E_{\tau}(0) \tau\left(A_{n}\right) E_{\tau}(0), A_{i} \in \mathfrak{A} \quad i=1,2 \ldots n
$$

it is only necessary to show that such elements may be weakly approximated by sequences. However it follows directly from Lemma 5 that they can even be approximated strongly.

We notice that this uniqueness proof does not depend upon a separability assumption for the representation induced by the state $\Phi$.

Now that we have seen that all invariant states over an asymptotically abelian algebra can be uniquely expressed in terms of invariant weakly clustering states we will denote those latter basic states by the special name $E$-states:

Definition 4. If $\mathfrak{A}$ is an asymptotically abelian algebra then the invariant weakly clustering states over $\mathfrak{A}$ are called $E$-states.

Theorem 1 has already shown that invariant weakly clustering states over a general algebra $\mathfrak{A}$ have a number of very special properties; the assumption of an asymptotically abelian character for $\mathfrak{A}$ increases this number considerably. The next theorem (a generalization of theorem 7 of $\mathrm{DKR}$ ) gives alternative properties which may be used to characterize $E$-states.

Theorem 3. Let $\Phi$ be an invariant state over an asymptotically abelian algebra $\mathfrak{A}$ and $\hat{\Phi}$ its canonical extension to $\mathfrak{A}_{1}^{T}$. If $(\pi, U)$ and $\hat{\pi}$ are the associated representations, on the Hilbert space $\mathfrak{H}$, of $(\mathfrak{A}, \mathbf{T})$ and $\mathfrak{A}_{1}^{T}$ and $\Omega$ is the corresponding invariant cyclic vector then the following are equivalent

i) $\Phi$ is a weakly clustering state,

ii) $\Omega$ is the only invariant vector in $\mathfrak{G}$, 
iii) $(\pi, U)$ is irreducible i.e. $\hat{\pi}$ is irreducible or $\hat{\Phi}$ is pure,

iv) $\hat{\pi}\left(\mathfrak{Q}_{1}^{T}\right)^{\prime \prime}$ is a factor i.e. $\hat{\Phi}$ is a primary state,

v) $\Phi(\hat{\Phi})$ is an extremal element of the convex set of invariant states over $\mathfrak{A}\left(\mathfrak{A}_{1}^{T}\right)$ i.e. $\Phi(\hat{\Phi})$ cannot be further decomposed in terms of invariant states,

vi) $\lim _{\alpha} \int d x f_{\alpha}(x)\left(\psi\left|U(x)-E_{\Omega}\right| \varphi\right)=0, \psi, \varphi \in \mathfrak{F}$,

vii) $\lim _{\alpha}\left\|\int d x f_{\alpha}(x)\left(U(x)-E_{\Omega}\right) \psi\right\|=, \psi \in \mathfrak{S}$,

viii) $\lim _{\alpha} \int d x f_{\alpha}(x)(\psi|\pi(A(x))-(\Omega|\pi(A)| \Omega)| \chi)=0, \psi, \chi \in \mathfrak{F}, A \in \mathfrak{A}$,

ix) $\lim _{\alpha}\left\|\int d x f_{\alpha}(x)(\pi(A(x))-(\Omega|\pi(A)| \Omega)) \psi\right\|=0, \quad \psi \in \mathfrak{H}, A \in \mathfrak{A}$.

In the above $f$ is any $M$-filter and $E_{\Omega}$ is the projector onto $\Omega$.

Proof. Most of the equivalences cited in the theorem have already been proved in the foregoing. In particular i) $\Leftrightarrow$ ii) $\Leftrightarrow$ vi) $\Leftrightarrow$ vii) $\Rightarrow$ iii) is given by theorem 1 , iii) $\Rightarrow$ iv) by definition and iv) $\Rightarrow$ iii) due to Corollary 2 of theorem 2. Further ix) $\Rightarrow$ viii) by definition and that viii) $\Rightarrow$ vi) is easily seen by choosing $\chi=\Omega$ and using the fact that $\Omega$ is cyclic for $\pi$. Next if $\hat{\Phi}$ is a pure state it is an extremal element of the set of all states and we clearly have iii) $\Rightarrow v$ ). We complete the proof of the theorem by demonstrating that $v) \Rightarrow$ iii) $\Rightarrow$ ii) and vii) $\Rightarrow$ ix).

$v) \Rightarrow$ iii) .If we assume that $\hat{\pi}$ is reducible we can decompose it into irreducibles and there will be a corresponding decomposition of $\hat{\Phi}$. However this latter decomposition will be a decomposition into invariant states because the commutant of $\hat{\pi}$ commutes with $U(x)$. Thus we have a contradiction.

iii) $\Rightarrow$ ii). Let us assume that there are many invariant states in $\mathfrak{F}$ and we denote the projector on all invariant states by $E(0)$. However due to lemma $4 E(0) \pi(\mathfrak{A}) E(0)$ is an abelian set and this is in contradiction with the irreducibility of $(\pi, U)$ unless $E(0)$ is one-dimensional i.e. $\Omega$ is unique.

vii) $\Rightarrow \mathrm{ix})$. We have straightforwardly that

$$
\begin{aligned}
\| \int d x f_{\alpha}(x)(\pi(A(x)) & -(\Omega|\pi(A)| \Omega)) \pi(B) \Omega- \\
& -\int d x f_{\alpha}(x) \pi(B)\left(U(x)-E_{\Omega}\right) \pi(A) \Omega \| \\
= & \left\|\int d x f_{\alpha}(x)[\pi(A(x)), \pi(B)] \Omega\right\| .
\end{aligned}
$$

However in the filter limit the right hand side tends to zero due to (31). Remembering that $\Omega$ is cyclic for $\pi$ and that all operators occurring in (62) are bounded we have immediately the complete equivalence vii) $\Leftrightarrow \mathrm{ix}$ ).

As we have now reduced the discussion of invariant states to a discussion of $E$-states it is perhaps at this point appropriate to comment upon the physical significance of these latter states in the light of Theorem 3. We would like particularly to comment upon condition viii) 12 Commun. math. Phys., Vol. 3 
of this theorem which shows that

$$
\lim _{\alpha} \int d x f_{\alpha}(x)(\psi|\pi(A(x))| \psi)=(\Omega|\pi(A)| \Omega),\|\psi\|=1
$$

Now if the $E$-state described an equilibrium situation in statistical mechanics and $\Omega$ was the vector representing the ground state of the system in equilibrium then $\psi$ would be a vector representing a state of the system which was locally disturbed from equilibrium. We then see from (63) that the average of all measurements of a local observable $A$ at the various points of space would be a global quantity whose value is independent of the vector state in which it is measured. Further from condition ix) we have

$$
\begin{aligned}
\lim _{\alpha} \int d x d y f_{\alpha}(x) f_{\alpha}(y)\{(\psi \mid \pi(A(x)) & \pi(A(y)) \mid \psi)- \\
& -(\psi|\pi(A(x))| \psi)(\psi|\pi(A(y))| \psi)\}=0
\end{aligned}
$$

for $A \in \mathfrak{A}, \psi \in \mathfrak{G}$ and $\|\psi\|=1$. This latter condition expresses the fact that the fluctuations of the values of the space averages of all local observables $A$ are small in all vector states, a characteristic of singlephase equilibrium.

Obviously the essential next step in an analysis of invariant states is to classify the $E$-states. One classification which appears to be of physical interest is based upon the analysis of the discrete spectrum of the spacetranslation operators. The necessary mathematical information concerning this spectrum is given by the following theorem.

Theorem 4. Let $\Phi$ be an $E$-state and $(\pi, U)$ the associated representations of $(\mathfrak{R}, \mathbf{T})$. The group representatives $U$ have the general spectral decomposition

$$
U(x)=\sum_{p_{n} \in \mathbf{S}_{D}} E\left(p_{n}\right) e^{i p_{n} x}+\int_{\mathbf{S}_{o}} d E(p) e^{i p x}
$$

where the sum and integral correspond to the discrete spectrum $\mathbf{S}_{D}$ and the continuous spectrum $\mathbf{S}_{C}$ of $U$ respectively. It follows that the discrete spectrum is subgroup i.e. if $p_{n}, p_{m} \in \mathbf{S}_{D}$ then $p_{n}+p_{m} \in \mathbf{S}_{D}$ and $-p_{n} \in \mathbf{S}_{D}$ and further it is simple i.e. for each $p_{n} \in \mathbf{S}_{D}$ the corresponding projector $E\left(p_{n}\right)$ is one-dimensional.

Proof. We have already seen that in an $E$-state $(\pi, U)$ is irreducible but from lemma 4 we know that $E\left(p_{n}\right) \pi(\mathfrak{A}) E\left(p_{n}\right)$ is abelian for $p_{n} \in \mathbf{S}_{D}$. Therefore we have a contradiction unless $E\left(p_{n}\right)$ is one-dimensional. This proves the simplicity of $\mathbf{S}_{D}$. Next we note that the eigenstate of $E\left(p_{n}\right)$ is cyclic for $(\pi, U)$, due to irreducibility, and hence cyclic for $\pi$. Now from (32) of lemma 4 we have, in particular, that

$$
E\left(p_{n}\right) \pi(A) E\left(p_{n}+p_{m}\right) \pi(B) E\left(p_{m}\right)=E\left(p_{n}\right) \pi(B) E(0) \pi(A) E\left(p_{m}\right) .
$$

For all $A, B \in \mathfrak{A}$. Now if we assume that $p_{n}, p_{m} \in \mathbf{S}_{D}$ but $p_{n}+p_{m} \notin \mathbf{S}_{D}$ we 
must conclude that

$$
E\left(p_{n}\right) \pi(B) E(0) \pi(A) E\left(p_{m}\right)=0
$$

for all $A, B \in \mathfrak{A}$. But as $E\left(p_{n}\right), E\left(p_{m}\right)$ project onto states cyclic under $\pi$ this would entail $E(0)=0$ which is a contradiction because $E(0)$ projects onto the invariant vector $\Omega$ associated with $\Phi$. This proves the additivity of $\mathfrak{S}_{D}$. The symmetry of $\mathbf{S}_{D}$ follows similarly from

$$
E(0) \pi(A) E\left(p_{n}\right) \pi(B) E(0)=E(0) \pi(B) E\left(-p_{n}\right) \pi(A) E(0)
$$

which is a special case of (32).

The result of the above theorem provides an immediate and natural classification of $E$-states for the case $\mathbf{T}=\mathbf{R}^{n}$.

Definition 5. If $\Phi$ is an $E$-state and $\Omega$ is the associated invariant vector and $U$ the corresponding representation of $\mathbf{T}$ then

1. $\Phi$ is called an $E_{\mathrm{I}}$-state if $\Omega$ is the only discrete eigenvector of $U$.

2. $\Phi$ is called an $E_{1 !}^{m}$-state if the discrete spectrum $\mathbf{S}_{D}$ of $U$ spans an $m$-dimensional subspace of $\mathbf{R}^{n}$ and if there exists a minimum nonzero distance between all pairs of points of $\mathbf{S}_{D}$.

3. $\Phi$ is called an $E_{\mathrm{III}}$-state if it does not fall into the two foregoing categories.

As $\mathbf{S}_{D}$ is additive the definition of $E_{\mathrm{II}^{-}}$-states is equivalent to the requirement that $\mathbf{S}_{D}$ contains no accumulation points. On the other hand in an $E_{\text {III }}$-state accumulation points of $\mathbf{S}_{D}$ will occur and due to the additivity property each point in some subset of $\mathbf{S}_{D}$ will be an accumulation point. Thus in $E_{1 I^{-}}$state $S_{D}$ will be dense in some subspace of $\mathbf{R}^{n}$ that it spans.

At this point we end our general analysis of $E_{\mathrm{I}}$-states and in the following section we will analyse $E_{\mathrm{II}^{\mathrm{I}}}$-states. We are unable to analyse

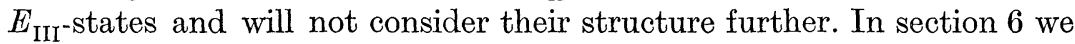
return a discussion of possible properties of $E_{\mathrm{I}}$-states.

\section{Lattice states}

The aim of this section is to present an analysis of $E_{\mathrm{II}^{-}}$-states. The major feature of our results is that an arbitrary $E_{\mathrm{II}}$-state can be uniquely decomposed into states which are invariant under a subgroup of translations and which are characterised by a number of equivalent conditions similar to those characterising $E_{\mathrm{I}}$-states. The methods which we use closely parallel those of previous sections; our general tactics are the following. For each $E_{\mathrm{II}}$-state we define a certain subgroup $\mathbf{T}_{L}$ of the group $\mathbf{T}$ ( $L$ for lattice). This subgroup consists of all translations which leave the corresponding discrete eigenvectors of $\mathbf{T}$ invariant. $\mathbf{T}_{L}$ acts as a group of automorphisms on the algebra $\mathfrak{A}$ and the $E_{\mathrm{II}^{-}}$state is an invariant state with respect to this group. Now we apply to the pair 
$\left(\mathfrak{A}, \mathbf{T}_{L}\right)$, or equivalently to $\mathfrak{A}_{1}^{\mathbf{T}_{L}}$ our previous results. As we mentioned earlier, these results are valid for any locally compact abelian group and are thus applicable to $\mathbf{T}_{L}$ with appropriate notational changes.

We begin by giving abstract definitions of $\mathbf{T}_{L}$ and related concepts and then illustrating these concepts by examples. Now the discrete spectrum $\mathbf{S}_{D}$ of $U(x)$ in an $E_{\mathrm{II}}$-state is, according to the definition, a discrete closed sub-group of the dual group $\tilde{\mathbf{T}}$ of $\mathbf{T}(\widetilde{\mathbf{T}} \equiv p$-space). We next define the closed subgroup $\mathbf{T}_{L}$ of $\mathbf{T}$ by the requirement that

$$
p_{n} x=0 \bmod 2 \pi, \quad p_{n} \in \mathbf{S}_{D} .
$$

This amounts to our prescription of selecting all translations which leave invariant the discrete eigenvectors of $U(x)$. The subgroup $\mathbf{T}_{L}$ is commonly referred to in the mathematical literature as the annihilator of $\mathbf{S}_{L} \subset \tilde{\mathbf{T}}$ (see [11] chapter 2,2-1). We next introduce the quotient group $\mathbf{K}=\mathbf{T} / \mathbf{T}_{L}$ $\mathbf{K}$ is the group of translations of $\mathbf{T}$ modulo translations of $\mathbf{T}_{L}$. Generally $\mathbf{T}_{L}$ and $\mathbf{K}$ can be identified with the dual groups of $\tilde{\mathbf{T}} / \mathbf{S}_{D}$ and $\mathbf{S}_{D}$ respectively ([11] theorem 2.1.2) and thus, since $\mathbf{S}_{D}$ is discrete, $\mathbf{K}$ is compact. The subgroup $\mathbf{T}_{L}$ will be discrete if, and only if, $\tilde{\mathbf{T}} / \mathbf{S}_{D}$ is compact i.e. if $\mathbf{S}_{D}$ spans $\widetilde{\mathbf{T}}$ as a vector space, which is the case of $E_{\mathrm{I}^{n}}^{n}$-states. In order to apply our previous results to the group $\mathbf{T}_{L}$ we must describe the Haar measure on $\mathbf{T}_{L}$. We obtain the simplest description for an $E_{I I}^{m}$-state in the following way. Let us call $\tilde{\mathbf{T}}^{m}$ the $m$-dimensional vectorial subspace spanned by $\mathbf{S}_{D}$ in $\widetilde{\mathbf{T}}$ and let us choose a complementary subspace $\widetilde{\mathbf{T}}^{n-m}$, then $\tilde{\mathbf{T}}$ is the product group $\widetilde{\mathbf{T}}^{m} \times \widetilde{\mathbf{T}}^{n-m}$. Consequently

$$
\tilde{\mathbf{T}} / \mathbf{S}_{D}=\tilde{\mathbf{T}}^{m} / \mathbf{S}_{D} \times \tilde{\mathbf{T}}^{n-m}
$$

where $\tilde{\mathbf{T}}^{m} / \mathbf{S}_{D}$ is compact and $\tilde{\mathbf{T}}^{n-m}$ is isomorphic to $\mathbf{R}^{n-m}$. Thus the dual group $\mathbf{T}_{L}$ of $\tilde{\mathbf{T}} / \mathbf{S}_{D}$ is equal to the product of the duals,

$$
\mathbf{T}_{L}=\mathbf{T}_{L}^{n} \times \mathbf{T}^{n-m}
$$

where $\mathbf{T}_{L}^{m}$ is a discrete group and $\mathbf{T}^{n-m}$ is again isomorphic to $\mathbf{R}^{n-m}$ ( $\mathbf{T}_{L}^{m}$ and $\mathbf{S}_{D}$ are "reciprocal lattices"). Thus the Haar measure on $\mathbf{T}_{L}$ is the product of a discrete sum over $\mathbf{T}_{L}^{m}$ and the Lebesgue measure on $\mathbf{T}^{n-m}$. In the event that $m=n\left(E_{\Pi 1}^{n}\right.$-states) $\mathbf{T}_{L}$ is discrete and the Haar measure is given by discrete sums.

Let us illustrate the above definitions in the case of two dimensions where we have the possibility of $E_{11}^{1}$-states or $E_{I I}^{2}$-states. Firstly we consider an $E_{\mathrm{II}}^{1}$-state where, by definition, the discrete spectrum $\mathbf{S}_{D}$ of $U$ consists of points equally spaced on a line. Choosing cartesian coordinates $\left(x_{1}, x_{2}\right)$ in $\mathbf{T}=\mathbf{R}^{2}$ and the corresponding coordinates $\left(p_{1}, p_{2}\right)$ in the dual space $\widetilde{\mathbf{T}}$ we can assume, without loss of generality, that the 
spectrum $\mathbf{S}_{D}$ consists of the points $\left(a_{1} N, 0\right)$ where $a_{1}$ is a fixed scale factor and $N$ takes integer values; in this case $\mathbf{T}_{L}^{1}$ is the discrete group of translations through distances $2 \pi M / a_{1}$ where $M$ takes integer values, in the $x_{1}$-direction. The group $\mathbf{T}_{L}$ consists of translations whose component in the $x_{1}$-direction is an integer multiple of $2 \pi / a_{1}$, and whose component in the $x_{2}$-direction is arbitrary. The quotient group $\mathbf{K}$ consists of translations in the $x_{1}$-direction modulo the translations of $\mathbf{T}_{L}^{1}$ and is isomorphic to the rotations (modulo $2 \pi$ ) on a circle. A general function $f$ on $\mathbf{T}_{L}$ can be viewed as a sequence of functions $f_{M}\left(x_{2}\right)$ and the integral of this function with respect to the Haar measure on $\mathbf{T}_{L}$ is given by

$$
\sum_{M=\infty}^{\infty} \int d x_{2} f_{M}\left(x_{2}\right) \text {. }
$$

Secondly if we consider an $E_{\mathrm{II}}^{2}$-state $\mathbf{T}_{L}$ will consist of translations of the type $\left(2 \pi M_{1} / a_{1}, 2 \pi M_{2} / a_{2}\right)$ where $a_{1}$ and $a_{2}$ are both fixed by the spectrum $\mathbf{S}_{D}$ and where $M_{1}, M_{2}$, take integer values. In this case $\mathbf{K}$ is isomorphic to the product of two groups both describing rotations (modulo $2 \pi$ ) on a circle. A function $f$ on $\mathbf{T}_{L}$ is then a double array of constants $f_{M_{1} M_{2}}$ and the integral of the function with respect to the Haar measure is the double sum

$$
\sum_{M_{1}}^{\infty} \sum_{M_{2}}^{\infty} f_{M_{1} M_{2}} .
$$

Now we proceed to the analysis of $E_{\mathrm{II}}$-states and we begin by introducing a number of notational definitions which will be used in the remainder of this section.

Definitions. Let $\Phi$ be an $E_{\mathrm{II}}$-state and $(\pi, U)$ the associated representations of $(\mathfrak{A}, \mathbf{T})$ on the space $\mathcal{F}$ with the cyclic invariant vector $\Omega$. We denote by $U_{L}$ the restriction of $U$ to the subgroup $\mathbf{T}_{L}$ of $\mathbf{T}$ and by $E$ the projector in $\mathfrak{G}$ on states invariant under $\mathbf{T}_{L}$ so that

and

$$
U_{L}\left(x_{L}\right)=E+\int d E_{L}(p) e^{i p x_{L}} \quad x_{L} \in \mathbf{T}_{L}
$$

$$
E=\sum_{p_{n} \in S_{D}} E\left(p_{n}\right)
$$

On the space $E \mathfrak{H}$ we define the algebra $\mathfrak{M}$ to be the $C^{*}$-algebra generated by $E \pi(\mathfrak{A}) E$ and we denote by $U_{E}$ the restriction of $U$ to $E \mathfrak{G}$. We note that for $x_{L} \in \mathbf{T}_{L}$ the restriction $U_{E}$ reduces to the identity (in $E \mathfrak{F}$ ) so that $U_{E}$ is in fact a representation of the compact quotient group $\mathbf{K}=\mathbf{T} / \mathbf{T}_{L}$.

With the above notation we now have

Lemma 6. The $C^{*}$-algebra $\mathfrak{M}$ is abelian and countably generated. Further, the set of operators $\mathfrak{W}$ and $U_{E}(\mathbf{K})$ is irreducible in $E \mathfrak{Y}$ and $\Omega$ is cyclic for $\mathfrak{M}$. 
Proof. As lemma 4 is valid for any locally compact abelian group we obtain immediately, by replacing $\mathbf{T}$ by $\mathbf{T}_{L}$ in that lemma, the fact that $\mathfrak{M}$ is abelian. As a consequence of (74) $\mathfrak{M}$ is generated by the $E\left(p_{n}\right) \pi(\mathfrak{Q}) E\left(p_{m}\right)$ but as $E\left(p_{n}\right)$ is one-dimensional for all $p_{n}$ we have that $\mathfrak{M}$ is countably generated. Finally since the set of operators $\pi(\mathfrak{Z})$ and $U$ are, by theorem 3 , irreducible in $\mathfrak{S}$ and $\Omega$ is cyclic for $\pi(\mathfrak{R})$ it follows that $\mathfrak{M}$ and $U_{E}(\mathbf{K})$ are irreducible in $E \mathfrak{G}$ and $\Omega$ is cyclic for $\mathfrak{M}$.

After these preliminaries we now wish to give the major result of this section concerning the decomposition of $E_{\mathrm{II}}$-states. It is a consequence of our earlier result, theorem 2, that there is a unique decomposition of an $E_{\mathrm{II}}$-state into states which are invariant with respect to $\mathbf{T}_{L}$ and weakly clustering with respect to this subgroup. As one would expect this decomposition 'breaks' the symmetry with respect to the group $\mathbf{K}$ and this is explicity displayed by

Theorem 5. If $\Phi$ is an $E_{\mathrm{II}}$-state then there is a unique decomposition of $\Phi$ into states $\Phi_{\xi}$ which are invariant and weakly clustering with respect to the subgroup $\mathbf{T}_{L}$. Furthermore this decomposition can be written as an integral with respect to the Haar measure $d m(\xi)$ of $\mathbf{K}$ and one has

$$
\Phi=\int_{\mathbf{K}} d m(\xi) \Phi_{\xi}
$$

in such a way that the states $\Phi_{\xi}$ are transforms of each other under the action of $\mathbf{K}$ i.e.

$$
\Phi_{\xi+\eta}(A)=\Phi_{\xi}(A(y)), \quad y \in \eta .
$$

The representations $\pi$ and $\pi_{\xi}$ associated with $\Phi$ and $\Phi_{\xi}$, respectively, are simultaneously faithful, but are inequivalent for $\xi \neq \eta$. However, the representations of $\mathbf{T}_{L}$ associated with the states $\Phi_{\xi}$ are all unitary equivalent.

Corresponding to the decomposition (75) there is a decomposition of the canonical extension $\hat{\Phi}$ of $\Phi$;

where

$$
\hat{\Phi}=\int_{\bar{K}} d m(\xi) \hat{\Phi}_{\xi}
$$

$$
\hat{\Phi}_{\xi+\eta}(X)=\hat{\Phi}_{\xi}(X(y)), \quad y \in \eta
$$

and the decomposition (77) is the central decomposition of $\hat{\Phi}$ over $\mathfrak{A}_{1}^{T_{L}}$ which is into pure states. The representations $\hat{\pi}$ and $\hat{\pi}_{\xi}$ of $\mathfrak{Q}_{1}^{T_{L}}$ generated by $\hat{\Phi}$ and $\hat{\Phi}_{\xi}$ are simultaneously faithful.

Proof. Before giving details we first outline the main features of the proof. Firstly we know from theorem 2 , on replacing $\mathbf{T}$ by $\mathbf{T}_{L}$, that there exists a unique decomposition of $\Phi$ into states which are weakly clustering and invariant with respect to $\mathbf{T}_{L}$. We will construct this decomposition by the "diagonalization" of the abelian algebra $\mathfrak{M}$ acting in $E \mathfrak{G}$ (a slight variant of the construction in the proof of theorem 2). Secondly we demonstrate that this decomposition can be rewritten in the form (75) 
by using the fact that the set of operators $\mathfrak{M} \cup U_{E}$ is irreducible in $E \mathfrak{G}$ and that $\mathbf{T}$ acts on $\mathfrak{M}$ in the covariant manner

$$
U_{E}(x) E \pi(A) E U_{E}(x)^{-1}=E \pi(A(x)) E .
$$

These facts, together with the remark that $\mathbf{T}_{L}$ is characterised as the set of $x \in \mathbf{T}$ acting trivially on $\mathfrak{M}$, allow us to establish an identification between the quotient group $\mathbf{K}=\mathbf{T} / \mathbf{T}_{L}$ and the spectrum $\subseteq$ of $\mathfrak{M}$. We now proceed to the details.

We recall that the abelian $C^{*}$-algebra $\mathfrak{M}$ can be identified with the set $\mathbf{C}_{0}(\mathbb{S})$ of continuous functions on $\mathbb{S}$ vanishing at infinity, through the well known Gelfand isomorphism defined by

$$
\hat{M}(s)=s(M)
$$

where $M \in \mathfrak{M}, s \in \mathbb{S}$ and $\hat{M} \in \mathbb{C}_{0}(\mathbb{E})$. The positive form on $\mathfrak{M}$ defined by $\Omega \in E \mathcal{G}$ is then represented by a Radon measure $m$ on $\subseteq$ i.e.

$$
(\Omega|M| \Omega)=\int d m(s) \hat{M}(s) .
$$

Since $\Omega$ is cyclic for $\mathfrak{M}$ in $E \mathfrak{F}$ the latter can be identified with the space $\mathbf{L}_{2}(\widetilde{S}, m)$ in such a way that if

$$
\psi \in E \mathfrak{G} \leftrightarrow \hat{\psi} \in \mathbf{L}_{2}(\Im, m)
$$

we have a "diagonalisation" of $\mathfrak{M}$ in the sense that (see [12] $\S 17.4$ )

$$
\widehat{M \psi}(s)=\hat{M}(s) \hat{\psi}(s), \quad \psi \in \mathfrak{G}, M \in \mathfrak{M} .
$$

This diagonalisation effects the decomposition of $\Phi$,

$$
\Phi(A)=(\Omega|E \pi(A) E| \Omega)=\int_{\mathcal{S}} d m(s) \Phi_{s}(A)
$$

where the states $\Phi_{s}$ are defined by

$$
\Phi_{s}(A)=s(E \pi(A) E)=\widehat{E \pi(A) E}(s)
$$

which implies that they are invariant and weakly clustering with respect to $\mathbf{T}_{L}$ through an argument identical to that given in the proof of theorem 2.

We next wish to examine the action of $U_{E}(x), x \in \mathbf{T}$, on $\mathbf{L}_{2}(\mathfrak{S}, m)$ $=E \mathfrak{G}$. From (79) we see that $\mathbf{T}$ acts on $\mathfrak{M}$ as a group of automorphisms

$$
M \in \mathfrak{M} \rightarrow M_{x} \in \mathfrak{M}
$$

of the algebra $\mathfrak{2}$. We define the action of $\boldsymbol{T}$ on the spectrum $\mathscr{\Xi}$ by

with

$$
s \in \mathfrak{S} \rightarrow s_{x} \in \mathfrak{S}
$$

so that

$$
s_{x}(M)=s\left(M_{-x}\right)
$$


From this we easily deduce the action of $U_{E}(x)$ on $\mathbf{L}_{2}(\mathfrak{S}, m)$ and we have

$$
\widehat{U_{E}(x) \psi}(s)=\hat{\psi}\left(s_{-x}\right)
$$

in accordance with (79). The fact that $U_{E}(x)$ is unitary reflects itself in the property that the measure $m$ is invariant for the mapping (87). Note that the mapping (87) is such that $(s, x) \rightarrow s_{x}$ is continuous form $\mathbb{\Xi} \times \mathbf{T}$ to $\mathbb{5}$. Indeed we have for arbitrary $M_{i} \in \mathfrak{M} i=1 \ldots n$

$$
\begin{aligned}
\left|s_{x}\left(M_{i}\right)-s_{x^{\prime}}^{\prime}\left(M_{i}\right)\right| & \leqq\left|s_{x}\left(M_{i}\right)-s_{x}^{\prime}\left(M_{i}\right)\right|+\left|s_{x}^{\prime}\left(M_{i}\right)-s_{x^{\prime}}^{\prime}\left(M_{i}\right)\right| \leqq \\
& \leqq\left|s\left(M_{i-x}\right)-s^{\prime}\left(M_{i-x}\right)\right|+\left\|M_{i-x}-M_{i-x^{\prime}}\right\|
\end{aligned}
$$

where

$$
\left|s\left(M_{i-x}\right)-s^{\prime}\left(M_{i-x}\right)\right| \leqq \frac{\varepsilon}{2} \quad i=1 \ldots n
$$

defines a weak neighbourhood of $s$ in $\mathcal{E}$ and

$$
\left\|M_{i-x}-M_{i-x^{\prime}}\right\| \leqq \frac{\varepsilon}{2}
$$

for $x-x^{\prime}$ small enough by our assumption (20).

Now let $u$ be an element of $\mathcal{S}$ and consider the orbit $\mathcal{O}_{u}$ of $u$ under $\mathbf{T}$ defined as the set

$$
\mathcal{O}_{u}=\left\{u_{x} ; x \in T\right\} \text {. }
$$

$\mathcal{O}_{u}$ is a Borel subset of $\mathbb{S}$ and, as a consequence of $(83)$ and $(90), \mathbf{L}_{2}\left(\mathcal{O}_{u}, m\right)$ is a subspace of $E \mathcal{F}$ invariant under the combined action of $\mathfrak{M}$ and $U_{E}$. Thus as the set of operators $\mathfrak{M} \cup U_{E}$ is irreducible $\mathbf{L}_{2}\left(\mathcal{O}_{u}, m\right)$ is either the null subspace or the whole $\mathbf{L}_{2}(\overleftarrow{ }, m)$ space and hence in the latter case the complementary set of $\mathcal{O}_{u}$ is of zero $m$-measure. Irreducibility implies that the invariant measure $m$ is ergodic and on the other hand $\mathfrak{M}$ is countably generated i.e. separable and consequently the spectrum $\mathfrak{S}$ admits a countable basis of open sets. Therefore ergodicity of $m$ implies transitivity (see [13], Theorem 3.9) and thus we can choose a $u \in \mathcal{S}$ such that $\mathcal{O}_{u}$ has an $m$-negligeable complement. Thus the integral in (87) can be equivalently taken over $\mathcal{O}_{u}$ i.e.

$$
\Phi(A)=\int_{\mathcal{O}_{u}} d m(s) \Phi_{s}(A)=\int_{\mathcal{O}_{u}} d m(s) s(E \pi(A) E)
$$

As the action of $\mathbf{T}$ on $\mathcal{O}_{u}$ is now strictly transitive we can, due to the continuity of the mapping $(s, x) \rightarrow s_{x}$, conclude that $\mathcal{O}_{u}$ is a homogeneous space for $\mathbf{T}$. Consequently, since $\mathbf{T}$ is a separable group and $\mathbf{T}_{L}$ is the stabilisier of the points in $\mathcal{O}_{u}$ i.e.

$$
\mathbf{T}_{L}=\left\{x \in T ; s_{x}=s\right\}, \quad s \in \mathcal{O}_{u}
$$

we have a homeomorphic mapping of $\mathcal{O}_{u}$ onto the quotient group $\mathbf{K}=\mathbf{T} / \mathbf{T}_{L}$. Explicitly, we have

$$
\xi \in \mathbf{K} \leftrightarrow u_{\xi} \in \mathcal{O}_{u}
$$


where $u_{\xi}$ is defined by

$$
u_{\xi}=u_{x} \quad \text { with } x \text { in the class } \xi \text { of } \mathbf{T} \text { modulo } \mathbf{T}_{L} .
$$

Furthermore the mapping is such that for $\eta, \xi \in \mathbf{K}$

$$
\eta+\xi \in \mathbf{K} \leftrightarrow u_{\eta+\xi} \in \mathcal{O}_{u}
$$

and the image of the measure $m$ on $\mathcal{O}_{u}$ is the Haar measure (which we also denote by $m$ ) on $\mathbf{K}$ because the Haar measure is the only quasiinvariant measure on $\mathbf{K}$.

We can now rewrite the decomposition (95) replacing $\mathcal{O}_{u}$ by the isomorphic $\mathbf{K}$

$$
\Phi(A)=\int_{\mathbf{K}} d m(\eta) \eta(E \pi(A) E)
$$

Now using the invariance of the Haar measure and taking account of (97) (99) we have

$$
\begin{aligned}
\Phi(A) & =\int_{\mathbf{K}} d m(\xi)(\xi+\eta)(E \pi(A) E) \\
& =\int_{\mathbf{K}} d m(\xi) \xi(E \pi(A(y)) E), \quad y \in \eta \\
& =\int_{\mathbf{K}} d m(\xi) \Phi_{\xi}(A)
\end{aligned}
$$

where the states $\Phi_{\xi}$ defined by

$$
\Phi_{\xi}(A)=\xi(E \pi(A(y) E), \quad y \in \eta
$$

obviously satisfy (76).

The above proof invoques general theorems of the theory of ergodic measures for the replacement of $\subseteq$ by $\mathbf{K}$ in the integral (84). In fact we can use the compactness of $\mathbf{K}=\mathbf{T} / \mathbf{T}_{L}$ to deduce in an elementary way the even stronger result that there exists a $u \in \mathbb{S}$ for which $\mathcal{O}_{u}$ is homeomorphic to $\mathbf{K}$ (without a zero-measure difference set). Since $\mathfrak{S}$ has a countable basis of open sets it is known that one can easily prove the existence of a $u \in \mathbb{S}$ such that the closure $\overline{\mathcal{O}}_{u}$ of $\mathcal{O}_{u}$ is the support of the ergodic measure $m$, i.e. coincides with the whole spectrum $\mathscr{\subseteq}$ since $\mathfrak{M}$ is by definition faithfully represented in $\mathbf{L}_{2}(\mathfrak{S}, m)$. The mapping of $\mathbf{K}$ onto $\mathcal{O}_{u}$ defined by (97) and (98) stems from the continuous mapping

$$
x \in \mathbf{T} \rightarrow u_{x} \in \mathcal{O}_{u}
$$

constant on the classes modulo $\mathbf{T}_{L}$ : it is therefore continuous from the compact space $\mathbf{K}$ to the Hausdorff space $\mathbb{S}$ and since it is one-to-one it is homeomorphic. We thus conclude that $\mathcal{O}_{u}=\overline{\mathcal{O}}_{u}=\mathfrak{S}$.

We now prove the rest of the theorem. Now that we have established (76) it is a direct consequence that $\pi_{\xi}$ and $\pi_{\eta}$ are simultaneously faithful. More generally (theorem 1.7 [14]) we have that

$$
\operatorname{Ker} \Phi=\bigcap_{\xi \in K} \operatorname{Ker} \Phi_{\xi} \text {. }
$$


The inequivalence of $\pi_{\xi}$ and $\pi_{\eta}$ follows as in theorem 2. To demonstrate the unitary equivalence of the representations of $\mathbf{T}_{L}$ associated with $\Phi_{\xi+\eta}$ and $\Phi_{\eta}$ we first note that the mapping

$$
\pi_{\xi+\eta}(A) \Omega_{\xi+\eta} \in \mathfrak{S}_{\xi+\eta} \rightarrow \pi_{\xi}(A(y)) \Omega_{\xi} \in \mathfrak{G}_{\xi} \quad y \in \eta
$$

extends to an isometric linear mapping of $\mathfrak{G}_{\xi+\eta}$ onto $\mathfrak{S}_{\xi}$ due to (76). The same equation also shows that

$$
\begin{aligned}
& \left(\pi_{\xi+\eta}(A) \Omega_{\xi+\eta}, U_{\xi+\eta}\left(x_{L}\right) \pi_{\xi+\eta}(B) \Omega_{\xi+\eta}\right) \\
& \quad=\left(\pi_{\xi}(A(y)) \Omega_{\xi}, U_{\xi}\left(x_{L}\right) \pi_{\xi}(B(y)) \Omega_{\xi}\right), \quad y \in \eta
\end{aligned}
$$

which demonstrates the unitary equivalence.

The properties of the decomposition of the canonical extension $\hat{\Phi}$ of $\Phi$ to $\mathfrak{A}_{1}^{T_{L}}$ follow immediately from theorem 2 and the above. Theorem 5 has shown that the representations $U_{\xi}$ of $\mathbf{T}_{L}$ associated with the states $\Phi_{\xi}$ occuring in the decomposition (75) are unitarily equivalent and we can also deduce the result,

Theorem 6. If $\Phi_{\xi}$ is a state occurring in the decomposition (75) of an $E_{\mathrm{II}}$-state and $U_{\xi}$ is the associated representation of $\mathbf{T}_{L}$ on $\mathfrak{G}_{\xi}$ then $U_{\xi}$ has one, and only one, discrete eigenvector in $\mathfrak{G}_{\xi}$. This unique eigenvector is the invariant vector $\Omega_{\xi}$ associated with $\Phi_{\xi}$.

Proof. Instead of considering the $\Phi_{\xi}$ we consider the canonical extensions over $\mathfrak{A}_{1}^{T_{L}}$ or rather their continuous extensions to the eveloping $C^{*}$-algebra $\overline{\mathfrak{2}_{1}^{T_{L}}}$ (see DKR Section 4 ). As $\hat{\Phi}_{\xi}$ is a pure state Kadison's transitivity theorem ([7] 2.8.4) assures us that any discrete eigenstate $\psi$ of $U_{\xi}\left(x_{L}\right)$ in $\mathfrak{G}_{\xi}$ with eigenvalue $e^{i q x_{L}}$ can be written

$$
\psi=\hat{\pi}_{\xi}(X) \Omega_{\xi}
$$

for some $\eta \in \overline{\mathfrak{2}_{1}^{T_{L}}}$. Therefore we have

$$
\hat{\Phi}_{\xi}\left(X^{*} X\left(x_{L}\right)\right)=\hat{\Phi}_{\xi}\left(X^{*} X\right) e^{i q x_{L}} .
$$

But from (78) we conclude that

$$
\begin{aligned}
\hat{\Phi}_{\xi+\eta}\left(X ^ { * } ( - y _ { \eta } ) X \left(-y_{\eta}\right.\right. & \left.\left.+x_{L}\right)\right) \\
& =\hat{\Phi}_{\xi+\eta}\left(X^{*}\left(-y_{\eta}\right) X\left(-y_{\eta}\right)\right) e^{i q x_{L}}, \quad y_{\eta} \in \mathfrak{S} \eta .
\end{aligned}
$$

This last relation shows that the vector

$$
\int_{K}^{\oplus} d m(\eta) \hat{\pi}_{\xi+\eta}\left(X\left(-y_{\eta}\right)\right) \Omega_{\xi+\eta}
$$

(where the choice of $y_{\eta} \in \mathfrak{S} \eta$ is a continuous section) in the direct integral space

$$
\int_{K}^{\oplus} d m(\eta) \mathfrak{S}_{\xi+\eta}
$$

is a discrete eigenstate of $U_{L}\left(x_{L}\right)$ with eigenvalue $e^{i q x_{L}}$ and we therefore 
conclude from (73) that $q=0$. Thus all discrete eigenvectors of $U_{\xi}$ are invariant vectors but as $\Phi_{\xi}$ is weakly clustering it follows from theorem 1 (applied with $\mathbf{T}_{L}$ ) that they all coincide with $\Omega_{\xi}$.

We note that the use of direct integrals in the above proof requires separability of the representation $\pi$ generated by the $E_{\mathrm{II}^{-}}$-state $\Phi$, however this restriction can probably be removed.

The study of $E_{\mathrm{II}}$-states has been reduced at this point to the study of states occurring in the decomposition (75) and from theorems 4 and 5 we see that these states have properties analogous to $E$-states. We will give them the special name $L$-states.

Definition 6. If $\mathfrak{A}$ is an asymptotically abelian algebra and $\mathbf{T}_{L}$ is a closed subgroup of the group $\mathbf{T}$ of space-translations then we call a state $\Phi$ over $\mathfrak{A}$ an L-state if $\Phi$ is invariant with respect to $\mathbf{T}_{L}$ and if the associated invariant vector $\Omega_{L}$ is the only discrete eigenstate of the corresponding representation $U_{L}$ of $\mathbf{T}_{L}$.

In analogy to the definition of $E_{\mathrm{I}}$-states we have in the above definition brought forward the fact that the discrete spectrum of $U_{L}$ is a point and the corresponding eigenvector unique. This implies, due to theorem 1 , that $L$-states are weakly clustering and in fact they enjoy all of the properties listed in Theorem 4.

Theorem 7. If $\Phi$ is an L-state then it has the equivalent properties i)-ix) of theorem 3 with the proviso that $\mathbf{T}$ should be replaced by $\mathbf{T}_{L}$ in the statement of these properties. (The replacement of $\mathbf{T}$ by $\mathbf{T}_{L}$ naturally entails replacing $d x$ by $d x_{L}$, the Haar measure on $\mathbf{T}_{L}$, and the interpretation of $f$ as an $M$-filter over $\mathbf{T}_{L}$ as described above.)

Proof. The proof of this theorem follows, in the same manner that its statement does, by replacing $\mathbf{T}$ by $\mathbf{T}_{L}$ in theorem 3 and the appropriate foregoing lemmas.

The result of this, and the previous, section have reduced the study of invariant states to the analysis of the very similar $E_{\mathrm{I}}$ and $L$-states together with an examination of $E_{\text {III }}$-states. In the following section we give a short discussion of properties, and conjectured properties, of the former states.

\section{6. $\boldsymbol{E}_{\mathbf{I}}$-states and $\boldsymbol{L}$-states}

As $E_{\mathrm{I}}$-states and $L$-states are so similar we will only discuss the former; to apply our discussion to the latter it is merely necessary to replace $\mathbf{T}$ by $\mathbf{T}_{L}$. In theorem 3 we showed that the weak and strong means of representatives $\pi(A(x))$ of $\mathfrak{A}$ in an $E$-state $\Phi$ are equal to $\Phi(A) I$ and our first remark is that the product means of $\pi\left(A_{1}\left(x_{1}\right)\right) \ldots \pi\left(A_{n}\left(x_{n}\right)\right)$ also exist in the weak and strong sense and are equal to $\Phi\left(A_{1}\right) \ldots \Phi\left(A_{n}\right) I$. This follows straightforwardly from lemma 3 which also precisely states what we understand by product means. Our next remark is that an 
$E_{\mathrm{I}}$-state, which is by definition weakly clustering, is possibly already strongly clustering. The representation $U$ of $\mathbf{T}$ associated with such a state has the form

$$
U(x)=E_{\Omega}+\int_{s_{L}} d E(p) e^{i p x}
$$

so the strong clustering property $(28)$ is equivalent to the condition

$$
\int_{\delta_{L}} d(\psi|E(p)| \varphi) e^{i p x} \underset{|x|=\infty}{\longrightarrow} 0
$$

on the continuous measure $d(\psi|E(p)| \varphi)$ where $\psi, \varphi$ are vectors in the representation space $\mathfrak{H}$. If this condition is not automatically fulfilled it should be a consequence of taking stronger 'local commutativity' conditions than (31) for the algebra $\mathfrak{A}$. The strong clustering property would be deducible if the representation $\pi(\mathfrak{U})$, associated with an $E_{\mathrm{I}}$-state, were a factor representation as the next theorem (essentially due to Araki [15] and Borchers [16]) shows.

Theorem 8. If $\Phi$ is a state (not necessarily invariant) over an asymptotically abelian algebra $\mathfrak{A}$ and if the associated representation $\pi$ of $\mathfrak{A}$ on $\mathfrak{G}$, is factorial then

$$
|\Phi(A(x) B)-\Phi(A(x)) \Phi(B)| \underset{|x|=\infty}{\longrightarrow} 0 .
$$

Proof. Firstly we note that $\Phi$ and $\pi$ are connected by

$$
\Phi(A)=(\xi|\pi(A)| \xi), \quad A \in \mathfrak{A}
$$

where $\xi \in \mathfrak{G}$ is cyclic for $\pi$. Next we define $\mathfrak{L}$ to be the $C^{*}$-algebra of operators generated by $\pi$ and its commutant $\pi^{\prime}$. With this definition we have firstly that

$$
\|[B, \pi(A(x))]\| \underset{|x|=\infty}{\longrightarrow} 0
$$

for each $A \in \mathfrak{Z}$ and $B \in \mathfrak{Q}$ due to asymptotical abelianness and secondly $\mathfrak{L}$ acts irreducibly in $\mathcal{S}$ due to $\pi$ being factorial i.e. $\left\{\pi^{\prime \prime} \cap \pi^{\prime}\right\}=\{\lambda I\}$.

However, due to this irreducibility we can use Kadison's transitivity theorem (see [7], 2.9.1) to conclude that every $B \in \mathcal{L}$ has a decomposition

such that

$$
B=(\xi|B| \xi)+B_{1}+B_{2}
$$

$$
B_{1}^{*} \xi=0, B_{2} \xi=0 \text { and } B_{1}, B_{2} \in \mathfrak{I} .
$$

In particular for $A, B \in \mathfrak{A}$

$$
\begin{aligned}
\Phi(A(x) B)-\Phi(A(x)) \Phi(B) & =\left(\xi\left|\pi(A(x)) B_{1}\right| \xi\right) \\
& =-\left(\xi\left|\left[\pi(A(x)), B_{1}\right]\right| \xi\right), \quad B_{1} \in \mathfrak{I} .
\end{aligned}
$$

Now (112) is an immediate consequence of (117) and (114).

We note that in the above theorem it is not necessary that there should be a unitary representation of $\mathbf{T}$ associated with $\Phi$. It is also 
interesting to note that the decomposition (115) is an abstract mathematical version of the splitting into "creation" and "annihilation" operators.

Let us now show that the converse of the above theorem is not true i.e. strong clustering does not imply that $\pi$ is factorial, and thus deduce that an $E_{\mathrm{I}}$-state is not necessarily a primary state over $\mathfrak{A}$. To demonstrate this we will consider an abelian algebra $\mathfrak{A}$ generated by unitary operators $U(f)$, acting on the Fock space $\mathfrak{H}_{F}$ with vacuum $\Omega$, where

and

$$
U(f)=\exp \left\{i\left(a(f)+a^{+}(f)\right)\right\} \quad f \text { real } \in \mathbf{L}_{2}(\mathbf{T})
$$

$$
\left[a(g), a^{+}(h)\right]=\int d x d y \overline{g(x)} h(y) \varrho(x-y) \quad g, h \in \mathbf{L}_{2}(\mathbf{T}) .
$$

For consistency we take $\varrho$ to be real and of positive type, now we have

$\left(\Omega\left|U(f) U\left(g_{z}\right)\right| \Omega\right)=\exp \left\{-\frac{1}{2} \int d x d y(f(x)+g(x-z)) \times\right.$

$$
\times(f(y)+g(y-z)) \varrho(x-y)\}
$$

where the action of $\mathbf{T}$ is

$$
g \rightarrow g_{z} ; g_{z}(x)=g(x-z) .
$$

It is straightforwardly checked from (118), and the definition of $\mathfrak{A}$ that, the state over $\mathfrak{A}$ is strongly clustering if we assume that

$$
p(x) \underset{|x|=\infty}{\longrightarrow} 0 \text {. }
$$

However (118) also shows that this state is not a primary state over $\mathfrak{A}$ because as $\mathfrak{A}$ is abelian a primary state is pure and hence multiplicative over the algebra.

We conclude from the above observations that in our general framework of asymptotic abelian algebras, which also includes abelian algebras, $E_{\mathrm{I}}$-states are not necessarily primary. However it is possible that they are strongly clustering.

\section{Conclusion}

In the present paper we have focused our attention on invariant states over an asymptotically abelian algebra as these states are of interest in statistical mechanics. We have shown how one is led to a study of particular "elementary" states and how states of a lower "crystalline" symmetry naturally arise through the breaking of translation symmetry.

The methods which we have developed have more general applications than those considered and it would be possible to analyse invariant states when the group $\mathbf{T}$ is replaced by a more general locally compact group $\mathbf{G}$. The decomposition theorem (theorem 2) hinges essentially on two results only 1) the weak mean of $U(x)$ is the projector $E(0)$, and 
2) the set $E(0) \pi() E(0)$ is abelian. The first of these results is dependent only on the existence of $M$-filters and the second then depends on having an appropriate asymptotic abelian condition. It is worth mentioning that even in the context of the present paper it is not necessary to have the asymptotic abelian property for all directions to obtain most of our results. If one had this property in all space-like directions, as in relativistic field theory, it would also be possible to obtain similar results.

A final remark is that the methods of mean values developed here might be used in relativistic field theory to replace the methods of big translations as has also been suggested privately by R. HAAG.

Acknowledgements. During the course of this work it was discovered that D. RUELLE was working on similar problems and the authors are grateful to him for a number of interesting and illuminating discussions which ensued and which affected the final form of the manuscript beneficially. We also acknowledge gratefully earlier discussions with $R$. HAAG which provided part of the impetus for this work and fruitful discussions with J. Dixmier, S. Doplicher, R. Kadison and N. Hugenholtz. The authors are indebted to the Ministère de l'Education Nationale, Paris, for granting one of them (D. W. R.) an exchange professorship which made their collaboration possible.

Note added in proof. The proof of the assertion in Lemma 5 that the strong means of elements of $\mathfrak{A}$ are weakly dense in $\beta_{\hat{r}}$ is not satisfactory. However this assertion is correct as will be shown at a later occasion.

\section{References}

[1] Doplicher, S., D. Kastler, and Derek W. Robinson: Covariance algebras in field theory and Statistical Mechanics, Preprint (1966).

[2] HaAG, R.: Nuovo Cimento 25, 281 (1962).

[3] Ruelle, D.: Cargèse Lecture Notes (1965).

[4] Araki, H., and E. J. Woods: J. Math. Phys. 1, 159 (1965).

[5] Robinson, D. W.: Commun. Math. Phys. 1, 159 (1965).

[6] RuElle, D.: States of physical systems. Preprint I.H.E.S. (1966).

[7] Dixmier, J.: Les $C^{*}$-algebres et leurs representations. Paris: Gauthier Villars 1964.

[8] HAAG, R., and D. KastLer: J. Math. Phys. 5, 848 (1964).

[9] SAKaI, S.: Trans. Am. Math. Soc. II 8, 406 (1965).

[10] Dixmier, J.: Les algèbres d'opérateurs dans l'espace hilbertien. Paris: Gauthier Villars 1957.

[11] Rudin, W.: Fourier analysis on groups. New York: Interscience Publishers 1962.

[12] Neumark, M. A.: Normierte Algebren. Berlin: V.E.B. Deutscher Verlag der Wissenschaften 1959.

[13] Mackey, G. W.: Theory of group representations. Mimeographed Chicago 1955.

[14] Fell, J. M. G.: Trans. Am. Math. Soc. 94, 365 (1960).

[15] Arakr, H.: On the algebra of all local observables. Prog. Theor. Phys. 32, 844 (1964).

[16] Borchers, H. J.: Commun. Math. Phys. 1, 57 (1965). 